\title{
Inhibition by stabilization: targeting the Plasmodium falciparum aldolase-TRAP complex
}

\author{
Sondra Maureen Nemetski ${ }^{1,9 \dagger}$, Timothy J Cardozo ${ }^{1,2^{*}}$, Gundula Bosch ${ }^{3,8}$, Ryan Weltzer ${ }^{4,8}$, Kevin O'Malley ${ }^{4,8}$, \\ Ijeoma Ejigiri ${ }^{5}$, Kota Arun Kumar ${ }^{6,10}$, Carlos A Buscaglia ${ }^{7}$, Victor Nussenzweig ${ }^{6}$, Photini Sinnis ${ }^{3,5,8}$, \\ Jelena Levitskaya ${ }^{3,8}$ and Jürgen Bosch ${ }^{4,8^{*}}$ (I)
}

\begin{abstract}
Background: Emerging resistance of the malaria parasite Plasmodium to current therapies underscores the critical importance of exploring novel strategies for disease eradication. Plasmodium species are obligate intracellular protozoan parasites. They rely on an unusual form of substrate-dependent motility for their migration on and across host-cell membranes and for host cell invasion. This peculiar motility mechanism is driven by the 'glideosome', an actin-myosin associated, macromolecular complex anchored to the inner membrane complex of the parasite. Myosin A, actin, aldolase, and thrombospondin-related anonymous protein (TRAP) constitute the molecular core of the glideosome in the sporozoite, the mosquito stage that brings the infection into mammals.
\end{abstract}

Methods: Virtual library screening of a large compound library against the PfAldolase-TRAP complex was used to identify candidate compounds that stabilize and prevent the disassembly of the glideosome. The mechanism of these compounds was confirmed by biochemical, biophysical and parasitological methods.

Results: A novel inhibitory effect on the parasite was achieved by stabilizing a protein-protein interaction within the glideosome components. Compound 24 disrupts the gliding and invasive capabilities of Plasmodium parasites in in vitro parasite assays. A high-resolution, ternary X-ray crystal structure of PfAldolase-TRAP in complex with compound 24 confirms the mode of interaction and serves as a platform for future ligand optimization.

Conclusion: This proof-of-concept study presents a novel approach to anti-malarial drug discovery and design. By strengthening a protein-protein interaction within the parasite, an avenue towards inhibiting a previously"undruggable" target is revealed and the motility motor responsible for successful invasion of host cells is rendered inactive. This study provides new insights into the malaria parasite cell invasion machinery and convincingly demonstrates that liver cell invasion is dramatically reduced by $95 \%$ in the presence of the small molecule stabilizer compound 24.

Keywords: Plasmodium falciparum, Glideosome, Drug discovery, Protein-protein interaction, Inhibitor, Stabilizer, Virtual library screening, X-ray crystal structure, Malaria

\section{Background}

Despite recent advances in treatment and prevention, malarial disease continues to afflict hundreds of millions of people every year, with growing resistance to current therapies [1-5]. Innovative treatments targeting

\footnotetext{
*Correspondence: Timothy.Cardozo@nyumc.org; jbosch@jhu.edu

†Sondra Maureen Nemetski and Timothy J Cardozo contributed equally

${ }^{2}$ Institute for Systems Genetics, New York University School of Medicine, New York, USA

8 Johns Hopkins Malaria Research Institute (JHMRI), Baltimore, USA

Full list of author information is available at the end of the article
}

hitherto under-exploited aspects of plasmodial biology are needed.

Plasmodium, as with other protozoan parasites belonging to the phylum Apicomplexa, progress through their life cycle by invading host cells. Gliding and active host cell invasion are thus crucial for these organisms, and are facilitated through an actin/ myosin motor complex located beneath the parasite's plasma membrane [6-8]. Herewith, the bridging enzyme PfAldolase, which binds actin in addition to its role in glycolysis [9], plays a key role: it connects the 
actin/myosin motor to trans-membrane adhesins of the thrombospondin-related anonymous protein (TRAP) family, which are expressed in a life-cycle stage specific manner [10]. Thus, during plasmodial liver, blood and transmission stages, PfAldolase binds the conserved C-termini of the plasmodial paralogs TRAP, MTRAP and CTRP, respectively [10-15] as well as other interaction partners such as the cytoplasmic tail of AMA-1 [16].

During the gliding and invasion processes, TRAP molecules are translocated from the anterior to the posterior end of the parasite, where they are cleaved within their transmembrane domain by a rhomboid protease $[17,18]$. The cleavage reaction leaves the extracellular TRAP domains bound to the host cell or substrate, while the cytoplasmic C-termini, also referred to as TRAP-tails, remain bound to PfAldolase, from which they are believed to dissociate and be recycled for degradation over time $[18,19]$. Un-liganded PfAldolase molecules are then available for binding other TRAP molecules, thus enabling continuous gliding motion on the host cell surface, which constitutes a necessary prerequisite for subsequent invasion [20]. A schematic overview of the glideosome components that were identified via combinatorial pull-down experiments in Plasmodium parasites [10] is given in Fig. 1a.

The study presented here aims to inhibit parasite motility and infectivity by targeting the aldolase-TRAP interaction within the glideosome. As an enzyme of the glycolytic pathway, however, aldolase is well conserved throughout all kingdoms (204/365 identical residues between human and Plasmodium aldolase, see Additional file 1). Inhibitor design targeting the PfAldolase molecule and its binding partners must therefore meet the challenge of avoiding cross-reactivity with human aldolase enzymes. This study employs a novel approach to rational drug design to meet this challenge.

While traditional, targeted inhibitor-design approaches are usually geared towards finding small molecules that prevent protein-protein interactions (PPI) critical to the pathogen [21], the hypothesis explored here is counter-intuitive: strengthening, instead of hindering, the PfAldolase-TRAP interaction is hypothesized to inhibit motility and invasion. The rationale for this counterintuitive approach is that the hybrid molecular surface formed partly by PfAldolase and partly by TRAP in the bound state of the complex is unique to the parasite and could allow Plasmodiumspecific targeting with small molecules that would not

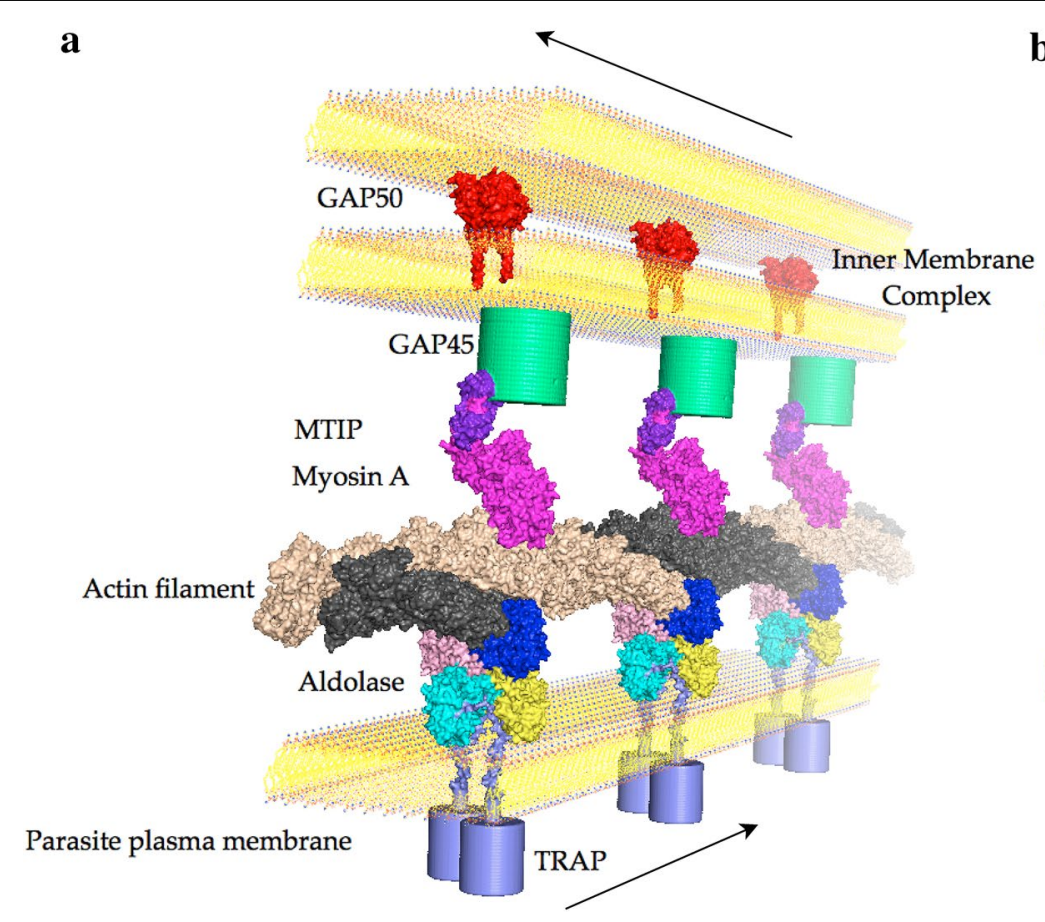

b
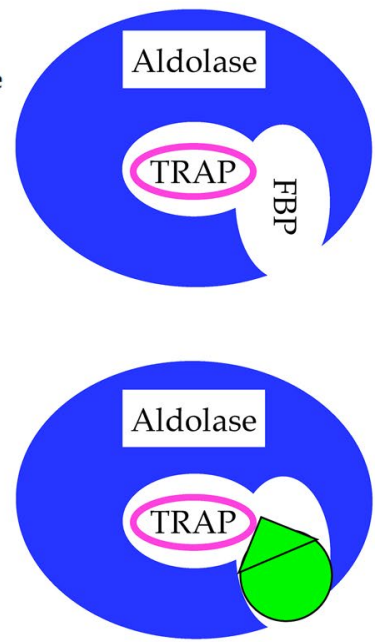

Fig. 1 Schematic overview of the glideosome components and principle of enzyme inhibition and complex stabilization. a The gliding machinery is anchored between the parasite plasma membrane and the inner membrane complex (IMC). Aldolase mediates a bridging function between short actin filaments and TRAP. b Simplified model of a single aldolase sub-unit with TRAP and F16P binding sites. TRAP-binding promoters (green) occlude access to the catalytic site of aldolase. 
cross-react with the human orthologues (Fig. 1b). More importantly, a fast dissociation of bound TRAP-tails (after protease cleavage) is critical for Plasmodium's ability to recycle PfAldolase molecules during gliding motility and host cell invasion $[17,18]$. Therefore, a small molecule designed to strengthen the PfAldolaseTRAP interaction should render this crucial dissociation process slow and inefficient, thus leading to an imbalance of this dynamic system and concomitant locomotion defects and likely reduced cell invasion. Equally importantly, the ternary complex of PfAldolase-TRAP and a stabilizing agent would be expected to interfere with glycolytic activity as the active site is occluded. It is unknown if the liver stage parasite relies on glycolytic activity for energy generation as does the blood stage form of the parasite [22]. However, PfAldolase is constitutively expressed in blood and gametocytes stages, suggesting it may be expressed, and is likely required, during liver stages as well (PlasmoDB PF3D7_1444800). A similar approach to stabilizing a PPI is described by Mecozzi et al. where they identified small molecules by virtual screening that were capable of stabilizing the Vps35-Vps26 interaction [23].

This hypothesis was tested using biophysical and biochemical assays as well as in vitro culture experiments with Plasmodium falciparum and Plasmodium berghei parasites to demonstrate that small molecules identified by virtual library screening (VLS) show an effect on gliding motility and hepatocyte invasion. A primary screen, which was comprised of VLS, PfAldolase catalytic activity, and thermal stability in the presence of small molecules, identified several compounds active in two or more assays. These were then further validated in two parasite specific assays, one investigating the impact on gliding motility and the second testing if parasites treated with small molecules are hindered in invasion of liver cells. Finally, the ternary co-crystal structure of PfAldolaseTRAP with compound 24 stabilizing the interaction was determined. The ternary complex is observed in all four copies of the PfAldolase tetramer represented in the crystal structure.

\section{Methods}

\section{Compound library and chemicals}

The screening library of 315,102 chemicals was provided as a structure description file (SDF) from the Chembridge Corporation (San Diego, CA, USA), as previously described. Unless otherwise noted, all compounds used in the in vitro and in vivo assays were obtained in powdered form from the Chembridge Corporation, and initially dissolved in $100 \%$ DMSO to obtain $100 \mathrm{mM}$ stock solutions, which were stored at $4{ }^{\circ} \mathrm{C}$ or $-20{ }^{\circ} \mathrm{C}$. Whenever possible, working dilutions in the relevant buffers were made within $24 \mathrm{~h}$ of the experiments in which they were used.

\section{Computational methods}

All computational work, including receptor modelling, VLS, docking, and hit-list post-processing was completed using tools in the ICM software suite produced by Molsoft, LLC (Version 3.7, La Jolla, CA, USA) with default parameters. VLS was performed as previously described [24]. The PocketFinder function of ICM was used to render solvent pockets suitable for small molecule ligand binding on the molecular surface of 3D structural protein models as previously described [25]. The crystal structure of the PfAldolase TRAP complex present in PDB ID 2pc4 was used as the starting point for all of the modelling, VLS and docking described in this study.

\section{Expression and purification of Plasmodium falciparum aldolase}

Cloning, expression, and purification of $P$. falciparum aldolase in Escherichia coli was performed using either of two previously described methods [26]. Prior to catalysis assays, the GST-tag was removed from the tagged protein using the Novagen Factor Xa Cleavage-Capture Kit according to manufacturer's instructions (EMD Biosciences, San Diego, CA, USA).

\section{Synthetic peptides}

Synthetic peptides derived from the cytoplasmic tails of $P$. falciparum and P. berghei TRAP were customsynthesized by Genemed Synthesis, Inc (TX, USA). These included PfTRAP25 (ETLGEEDKDLDEPEQ FRLPEENEWN), PfTRAP6 (EENEWN), PbTRAP25 (VMADDEKGIVEDEGFKLPEDNDWN), and PbTRAP6 (EDNDWN).

\section{Thermal shift assay}

The results reported here measured the effect of the VLS hits on a complex of recombinant $P$. falciparum aldolase and the PbTRAP6 peptide described above. The assays were conducted and analysed as per previously published protocols in triplicates $[27,28]$.

\section{Aldolase catalysis assay}

The protocol utilized here was based on that provided by Sigma-Aldrich ${ }^{\circledR}$ (St. Louis, MO, USA), and all of the reagents (listed below) other than drugs, TRAP and aldolase were obtained from that company as well. Briefly, aldolase was pre-incubated for $10 \mathrm{~min},+/-$ TRAP peptide (PfTRAP25, described above), +/- compound or DMSO at room temperature. The other reagents $(\alpha-G D H / T P I$, $\beta$-NADH, F16P in order) were then added, yielding a final reaction mixture containing 0.02 units $/ \mathrm{ml}$ aldolase 
( 1 unit $=$ amount of aldolase required to convert $1 \mu \mathrm{M}$ of F16P to DHAP and G3P per minute at $\mathrm{pH} 7.4$ and $25^{\circ} \mathrm{C}$; for these studies, this usually amounted to $\sim 50 \mathrm{nM}$ aldolase, based on an estimated purification yield of 10 units/ $\mathrm{mg}$ aldolase), $2 \mathrm{mM}$ F16P, $0.13 \mathrm{mM} \beta-\mathrm{NADH}, 2$ units/ $\mathrm{ml} \alpha-\mathrm{GDH} / \mathrm{TPI}(1$ unit $=$ amount of $\alpha \mathrm{GDH}$ required to convert $1.0 \mu \mathrm{M}$ of DHAP to $\alpha$-glycerophosphate per min at $\mathrm{pH} 7.4$ and $25{ }^{\circ} \mathrm{C}$ ), $100 \mathrm{nM}$ TRAP (or DMSO), and 5-100 $\mu \mathrm{M}$ compound (or DMSO) in catalysis buffer (0.2 M glycine titrated to $\mathrm{pH} 7.3$ with Trizma Base). A buffer with low ionic strength was used to avoid interference with the electrostatic interactions between aldolase and TRAP. Reactions were carried out either in a final volume of $1 \mathrm{ml}$, in standard plastic cuvettes (assays with PfTRAP25), or in a 96-well format with $100 \mu$ l reaction volume (assays with PbTRAP6). NADH consumption was measured at $340 \mathrm{~nm}$ for $10 \mathrm{~min}$ at $25^{\circ} \mathrm{C}$, using a SpectraMax M2e Microplate Reader (Molecular Devices, Sunnyvale, CA, USA). As many of the compounds had measurable inherent absorbance at $340 \mathrm{~nm}$, the baseline absorbance of each compound when dissolved in catalysis buffer at the tested concentration was measured and subtracted from the values obtained during the kinetic run. Suramin, a known aldolase inhibitor, was used as a positive control for aldolase inhibition [29].

To test if compound 24 had an inhibitory effect on human aldolase, rabbit muscle aldolase was used, which is $99.3 \%$ sequence identical to human aldolase. The assay was performed as described previously, however in the absence of TRAP-peptide to identify if the compound inhibited catalytic activity by itself.

\section{X-ray crystallography}

Compounds were co-crystallized with the PbTRAP6 peptide and purified $P$. falciparum aldolase as per previously published protocols [26]. Crystallization trials of PfAldolase with TRAP in the presence of different concentrations of the small molecules were set up at $20^{\circ} \mathrm{C}$. In most cases crystals of different sizes appeared within 1 week, many of the small molecules resulted in precipitated solutions at higher concentrations. Multiple synchrotron datasets were collected from crystals grown in the presence of 2 mM compounds $1,2,3,5,6,8,11,12,13,21,24,30,42$, 43,49 , and 54, resulting in diffraction from 2.1 to $4 \AA$ A resolution. Data processing and scaling was carried out with XDS/XSCALE [30]. The scaled data were then subjected to molecular replacement with the coordinates of $2 \mathrm{pc} 4$ [26] followed by automatic refinement using the Phenix suite [31]. All coordinates were refined below an $R_{\text {work }} / R_{\text {free }}$ of 28/32 prior to inspection of the electron density maps. Every dataset was visually inspected using Coot [32] and small molecule ligands were searched for either automatically using the find unmodelled blob function or manually by inspecting the four TRAP binding sites of each aldolase subunit near residues R48 and R309. The ternary complex of PfAldolase-TRAP with compound 24 was refined to an $\mathrm{R}_{\text {work }} / \mathrm{R}_{\text {free }}$ of 18.7/24.8 with one Ramachandran outlier as reported by Molprobity [33]. Compound 24 ligand occupancy was automatically refined using Phenix refine to $0.81-0.87$, indicating a high occupancy of the compound in the four binding sites. A summary of the data reduction and refinement statistics are provided in Table 1. The structure factors and coordinates of the final model have been deposited with the PDB under accession code 4TR9.

\section{Surface plasmon resonance assay}

A CM5 chip was prepared and conjugated with Neutravidin, allowing the subsequent capturing of biotinylated peptides, as described in [34]. All experiments were

Table 1 Data collection and refinement statistics for PDB entry 4TR9

\begin{tabular}{|c|c|}
\hline Beam line & SSRL 12-2, micro focus \\
\hline Wavelength $(\AA)$ & 0.9795 \\
\hline Resolution range $(\AA)$ & $44.85-2.11(2.19-2.11)$ \\
\hline Space group & $P 2_{1} 2_{1} 2_{1}$ \\
\hline Unit cell $\left(\AA^{\circ}{ }^{\circ}\right)$ & 69.89139 .56142 .10909090 \\
\hline Total reflections & $281,841(1,566)$ \\
\hline Unique reflections & $49,244(642)$ \\
\hline Multiplicity & $5.7(2.4)$ \\
\hline Completeness (\%) & $61.09(8.08)$ \\
\hline Mean I/sigma (I) & $21.47(1.04)$ \\
\hline Wilson B-factor & 36.99 \\
\hline R-merge & $0.0702(0.91)$ \\
\hline R-meas & 0.077 \\
\hline $\mathrm{CC} 1 / 2$ & $0.999(0.654)$ \\
\hline$C C^{*}$ & $1(0.889)$ \\
\hline R-work & $0.187(0.282)$ \\
\hline R-free & $0.248(0.289)$ \\
\hline Number of non-hydrogen atoms & 11,395 \\
\hline Macromolecules & 10,889 \\
\hline Ligands & 84 \\
\hline Water & 422 \\
\hline Protein residues & 1,416 \\
\hline RMS (bonds) & 0.010 \\
\hline RMS (angles) & 1.38 \\
\hline Ramachandran favoured (\%) & 94 \\
\hline Ramachandran outliers (\%) & 1 \\
\hline Clashscore & 8.58 \\
\hline Average B-factor & 46.50 \\
\hline Macromolecules & 46.30 \\
\hline Ligands & 59.70 \\
\hline Solvent & 48.30 \\
\hline
\end{tabular}

Statistics for the highest-resolution shell are shown in parentheses. 
carried out at $25{ }^{\circ} \mathrm{C}$ using a running buffer consisting of $10 \mathrm{mM}$ Hepes pH 7.5, $150 \mathrm{mM} \mathrm{NaCl}, 1 \mathrm{mM} \mathrm{MgCl}, 0.2 \%$ Tween 20, 1 \% DMSO. Purified PfAldolase was passed over a reference flow cell as well as over a PfTRAP-, PvTRAP- and PfMTRAP-tail exposing surface. Binding of PfAldolase was measured in the presence of different concentrations ranging from 125 to $1,000 \mu \mathrm{M}$ of compound 24. All measurements were performed in triplicates interspersed by blank injections. Data analysis was carried out with Scrubber (BioLogic Software) using double referencing method and correcting for DMSO absorption effects.

\section{Hepatocyte viability assay}

The VLS hits were screened for their affects on cultured HC-04 hepatocytes (ATCC, Manassas, VA, USA) as previously described [35-37]. To determine toxicity of compounds 1, 3, 24, 42 and 43 on human hepatocytes, human hepatocyte cell line HC-04 capable of supporting P. falciparum development in vitro [38] was exposed to $1 \mathrm{mM}$ of each compound for $96 \mathrm{~h}$ followed by Annexin V-APC and Propidium Iodide staining done according to manufacturer's instructions (Apoptosis Detection Kit, eBioscience Inc, San Diego, CA, USA). Samples were analysed using flow cytometry (FACS-Scan, BD Biosciences) and the percentage of Annexin $\mathrm{V}$ negative/Propidium Iodide negative viable cells was calculated using FlowJo analysis software (Tree Star Inc, Ashland, OR, USA).

\section{Sporozoite motility assay}

Compounds were tested for their effect on $P$. berghei sporozoite motility using established protocols [39, 40] For the assays described here, sporozoites were pre-incubated with each compound at $500 \mu \mathrm{M}$ for $10 \mathrm{~min}$ at $28{ }^{\circ} \mathrm{C}$ and the sporozoites remained in the presence of the compound (or DMSO) during the $1 \mathrm{~h}$-long assay at $37^{\circ} \mathrm{C}$. The quantity of motile parasites, and the numbers of their trails were then calculated to assess the compounds' effects.

\section{Sporozoite invasion assay}

The sporozoite neutralization assay was carried out as previously described [41]. Briefly, P. berghei sporozoites were pre-incubated with $500 \mu \mathrm{M}$ of the drugs or DMSO, and then allowed to infect human HepG2 cells (ATCC Collection). The HepG2 cells were collected after $40 \mathrm{~h}$, and the infectivity of the parasites was quantified by realtime PCR using primers specific for the P. berghei $18 \mathrm{~S}$ rRNA [42].

\section{Results}

Identification of ligand-accessible pockets through VLS

Small molecules with the potential to stabilize the interaction of the hybrid interface of TRAP with PfAldolase were identified by VLS on a previously described co-crystal structure of $P$. falciparum aldolase in complex with a short cytoplasmic tail of TRAP [26] (Fig. 1b). A necessary feature for targeting a particular molecular surface with VLS is the presence of an optimally located, appropriately sized 'druggable pocket' (i.e., a ligand-accessible cavity or surface) against which to screen a chemical library [43]. Suitable pockets in the PfAldolase-TRAP target (PDB code 2pc4, 2.4 A resolution, $R_{\text {work }} / R_{\text {free }}=20.1 / 25.0$ ) [26] were located with the ICM PocketFinder [25] (Molsoft, LLC., La Jolla, CA, USA), setting pocket form and identity as key strategic parameters. For the present experimental design, the pocket walls needed to be formed by the mixed surface of both TRAP and PfAldolase (Fig. 1b). As shown in Fig. 2a, b and Additional file 1, three pockets
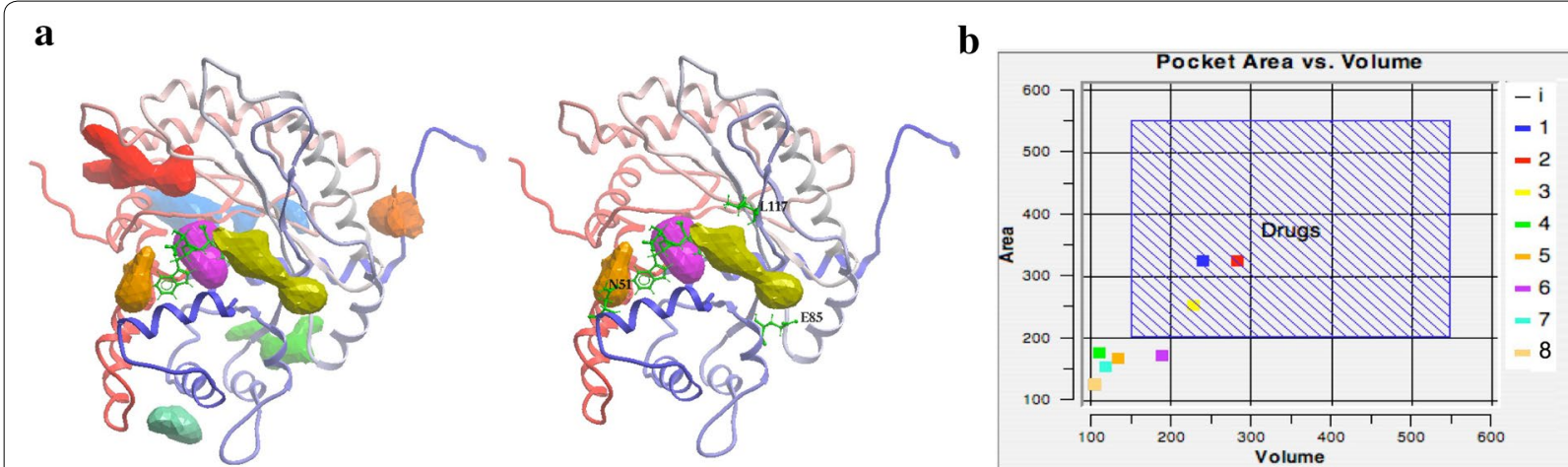

Fig. 2 Overview of the PfAldolase-TRAP druggable pockets. a Potential ligand-binding pockets in PfAldolase. Eight pockets were identified in the cocrystal structure of aldolase bound to TRAP (PDB ID: 2pc4 [26]). Aldolase is shown as a ribbon coloured in a smooth gradient from blue at its $\mathrm{N}$-terminus to red at its C-terminus. TRAP is shown as a green ball-and-stick model. Only pockets 3, 6 and 8 are displayed because these three were targeted by the design with VLS since they are contacted by three non-conserved residues within aldolase-N51, E85, L117 ( $g r e e n$ ballsand-sticks) — as well as by the TRAP peptide. b Plot of the surface area $\left(\AA^{2}\right)$ vs volume $\left(\AA^{3}\right)$ for the eight pockets. Pockets 1,2 and 3 fall within the $100-500 \AA^{2 / 3}$ area/volume range, which is the usual area/volume for most FDA-approved drugs, following Lipinski's rule of five [70]. 
(coloured yellow, purple, and orange, respectively) met this criteria. These pockets juxtapose with the TRAP binding site, contacting the non-conserved PfAldolase residues, N51, E85 and L117, as well as TRAP itself (balls-and-sticks in the Figure). While two of the pockets (purple and orange in Fig. 2a, b) individually have smaller-than-desirable area-to-volume ratios for drug binding [43], compound fragments fitting them could still be useful for a fragment-based approach to the design of the sought-after drug. Additionally, their key locations within the target region justified including them in the target VLS area.

\section{Target site modelling}

The precise conformation of the target pocket may strongly influence the selection of compounds by the VLS algorithm, as the target site is not flexible during the docking procedure. Therefore, in addition to screening the co-crystal structure of $P$. falciparum aldolase bound to a hexapeptide derived from the $\mathrm{C}$-terminus of P. berghei TRAP6 (PDB ID 2pc4, '2pc4 model'), additional screens were carried out against two additional models of the complex generated in silico: one in which the $P$. berghei TRAP sequence (EDNDWN) was modified to its $P$. falciparum counterpart (EENEWN, 'falciparum model'), and one in which the final TRAP residue was modified to alanine (EENDWA), in order to simulate induced fit via the 'gapped-pocket' method ('gappedpocket model') [44]. The different VLS receptor models and the areas in which the docking was concentrated are shown in Additional files 2 and 3.

\section{Virtual hit group selection through target site docking}

315,102 small molecules, representing a sub-set of the ChemBridge ${ }^{\circledR}$ hit2lead database (San Diego, CA, USA), were docked to the three different conformations of the target site using the ICM-VLS algorithm (Molsoft LLC, La Jolla, CA, USA). Three independent virtual screens against each receptor model, specifically targeting the PfAldolase-TRAP interface and surrounding residues, yielded 182 unique hits. To further narrow this preselection, these 182 compounds were re-docked to their respective receptors using the slower, more energetically accurate ICM-DOCK algorithm (Molsoft LLC, La Jolla, CA, USA). This step eliminated hits whose re-docked poses and/or energy scores differed significantly from the initial VLS results, as well as those with predicted lipophilicity (partition coefficients; cLogP) $<-2$ or $>4.60$ of the 69 remaining compounds were then purchased from ChemBridge (San Diego, CA, USA) for in vitro and cellbased assay testing. The individual small molecule structures as well as details of the VLS and docking results for these 60 compounds are listed in Additional file 4.

\section{In vitro hit validation by enzymatic activity and thermal stability assays}

In keeping with the novel hypothesis presented here, the initial 60 docking hits were subjected to biochemical and biophysical tests to identify those small molecules that would actually enhance the PfAldolase-TRAP interaction and thereby occlude access of fructose 1,6-bisphosphate as a substrate to the active site of the enzyme (Fig. 1b). As mentioned above, TRAP binding has an inhibitory effect on aldolase's glycolytic activity as previously demonstrated [26] (Fig. 1b).

To this end, the effects of the 60 pre-selected VLS hit compounds on PfAldolase's enzyme activity were first investigated in the presence and absence of TRAP. TRAP is a competitive inhibitor of PfAldolase as the binding sites of the glycolytic substrate fructose 1,6-bisphosphate (F16BP) and TRAP partially overlap [26]. Following the method of Döbeli et al. [22], PfAldolase enzyme activity was assayed by monitoring NADH consumption. The presence of the inhibitor TRAP expectedly caused a dose-dependent decrease in NADH consumption as previously published [26]. Hit compounds were considered potential stabilizers of the PfAldolase-TRAP interaction if they promoted an additional decrease in $\mathrm{NADH}$ consumption/PfAldolase activity in the presence versus absence of TRAP. The change in the $V_{\text {max }}$-rate compared to the attenuated $P f$ Aldolase-TRAP control distinguishes between inhibitory molecules with a negative $\Delta V_{\max }$ and TRAP-displacing molecules with a positive $\Delta \mathrm{V}_{\max }$ (Fig. 3a). TRAP-displacing molecules would likely interfere with human aldolase as the residues to which TRAP binds are identical between human and Plasmodium aldolase and are therefore undesired hits [26]. In order to identify sequence-specific differences in compound binding, all 60 VLS hits were assayed independently against PfAldolase with peptides derived from either $P$. berghei (DWA) or P. falciparum TRAP (DWN).

Furthermore, the thermal stability [27] of the PfAldolase-TRAP complex was investigated with or without enzyme inhibition promoting hit compounds, in order to exclude effects resulting merely from structural destabilization due to compound addition. The melting temperature $\left(\mathrm{T}_{\mathrm{M}}\right)$ of the PfAldolase-TRAP complex was assayed in the presence versus absence of hit compounds, where a strongly negative $\mathrm{T}_{\mathrm{M}}$-shift would indicate a destabilizing, denaturing effect. However, compounds causing only marginally negative $T_{M}$ 's or positive $T_{M}$ 's shifts compared to the PfAldolase-TRAP control were considered as potentially viable hits for further analysis (Fig. 3b, c).

At $100 \mu \mathrm{M}$ final concentration of the compounds, 13 (compounds 3, 5, 14, 15, 18, 25, 26, 28, 33, 42, 43, 47, and 48) produced a positive shift of $>2{ }^{\circ} \mathrm{C}$ in the melting point of a complex of recombinant $P$. falciparum 

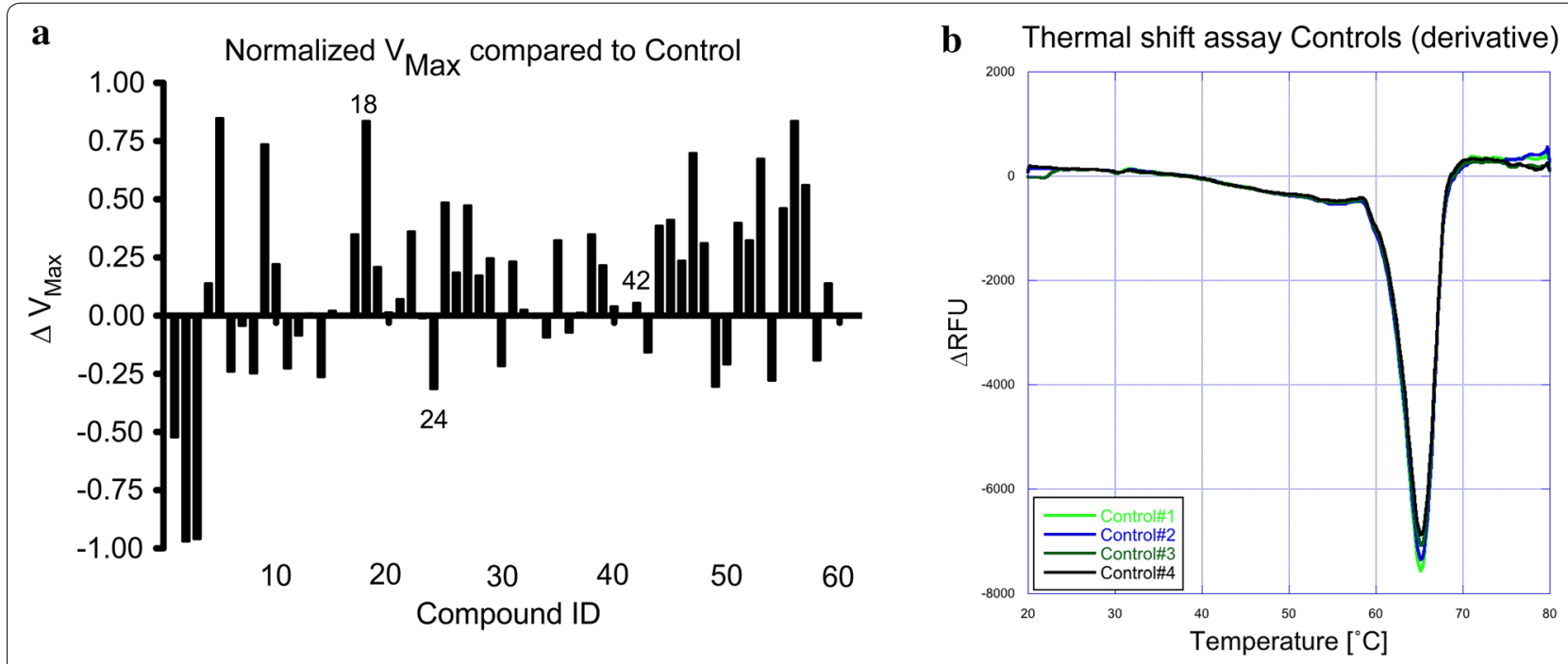

c $\mathrm{T}_{\mathrm{M}}$ PfAldolase+TRAP+Compound
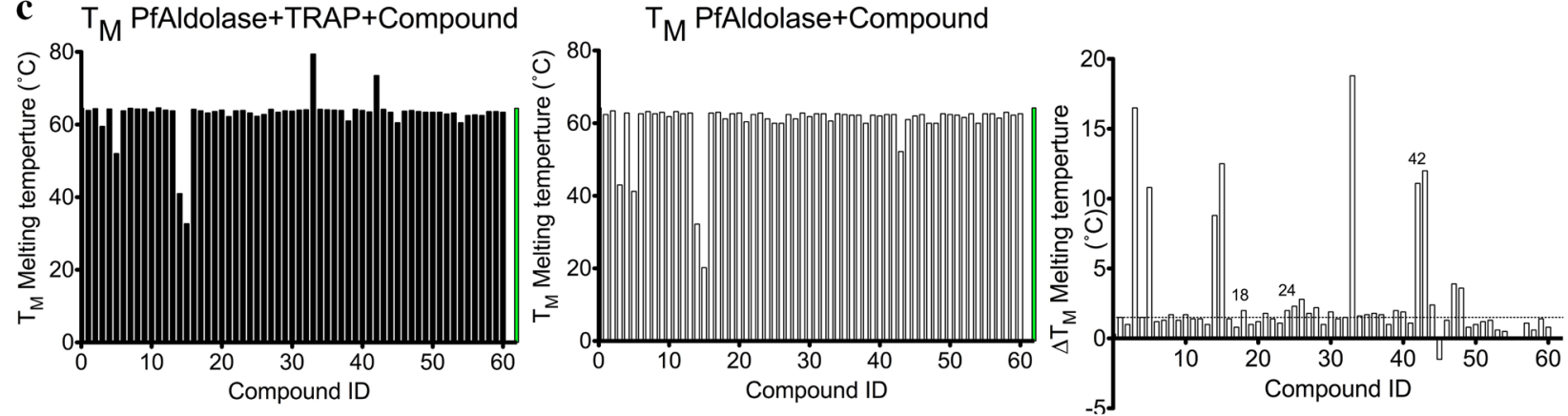

Fig. 3 PfAldolase catalytic activity and thermal stability in the presence of compounds. a Normalized bar graph representing the change in $\Delta V_{\max }$ relative to the control in the presence of each of the 60 ordered compounds. All assays were carried out in the presence of TRAP as used for the reference control. A negative $\Delta \mathrm{V}_{\text {max }}$ indicates further inhibition of glycolysis in the presence of the compound compared to PfAldolase with TRAP only. A positive $\Delta \mathrm{V}_{\max }$ indicates displacement of TRAP by the compound resulting in an increased catalytic activity. Compounds inhibiting invasion in an in vitro culture assay are numbered in the plot (see Fig. 5). b Thermal stability assay derivative curves of four controls of PfAldolase with PfTRAP. The $T_{M}$ is indicted by the minima of the derivative at approximately $65^{\circ} \mathrm{C}$. c Average of a triplicate thermal stability assay in the presence of small molecules with and without PfTRAP peptide. The green bar represents the melting temperature of the control without compound. The change in thermal stability $\left(\Delta T_{M}\right)$ in the presence of PfTRAP per compound is depicted in the last graph. The dotted line represents the cutoff of a shift greater than $1.5^{\circ} \mathrm{C}$.

aldolase and the same $P$. berghei TRAP hexapeptide used to solve the TRAP-aldolase co-crystal structure, suggesting a stabilizing effect (Fig. 3b, c). Interestingly, five compounds produced a negative $\mathrm{T}_{M}$ shift of $>2{ }^{\circ} \mathrm{C}$ compared to the control (compounds 3, 5, 14, 15, 43) when no TRAP-peptide was present, some of which could then be stabilized when TRAP was added (Fig. 3c, third panel).

Preliminary ligand based SAR analysis after primary screen Many of the VLS hits share similar chemical scaffolds (Fig. 4; Additional file 4). In particular, many of the compounds that were active in the catalysis or thermal shift assays, including compounds $1,9,13,16,17,19$, and 21 , as well as compounds 18, 24 and 42, which were also active in in vitro motility and cell invasion assays (see below), contained $N$-(benzylideneamino)benzamide (Fig. 4b). The docking results and the crystallographic data for the active compounds suggest that several of these hits also make similar hydrogen-bonding and electrostatic contacts with the PfAldolase-TRAP complex via functional groups extending off of the scaffold's two benzene rings (Additional file 5).

In vitro parasite hit validation by gliding motility and liver cell invasion assays

Twelve of the biochemically-validated compounds were assayed for their effects on parasite motility and infectivity. As shown in Fig. 5 and Additional file 6, two of the compounds, 24 and 42 , had a pronounced effect on 


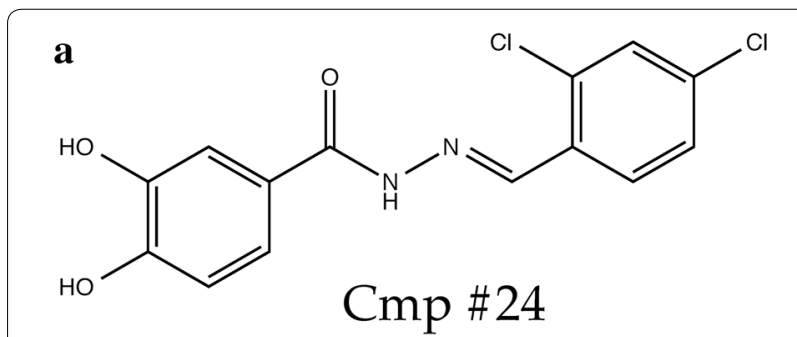<smiles>O=C(N/N=C/c1ccccc1O)c1ccc(O)c(O)c1</smiles><smiles>COc1cc(C(=O)N/N=C/c2cc(Cl)ccc2O)ccc1O</smiles>

b<smiles>O=C(N/N=C/c1ccccc1)c1ccccc1</smiles>

Fig. 4 Identified scaffold and chemical formulas. a Chemical formulas of compounds 18,24 and 42 showing a phenotypic effect on parasite gliding and parasite invasion of liver cells. b Frequently observed common scaffold of our VLS hits was a N-(benzylideneamino) benzamide ring system.

gliding motility when assayed at $500 \mu \mathrm{M}$ against isolated P. berghei sporozoites.

These same compounds, as well as compound 18, impaired the ability of parasites to invade hepatocytes when tested at $500 \mu \mathrm{M}$ in a sporozoite neutralization assay [41, 42]. As shown in Fig. 5 and Additional file 6, compounds 18, 24 and 42 produced 58, 95 and $34 \%$ inhibition of hepatocyte invasion, respectively as assayed by RT-PCR of $P$. berghei $18 \mathrm{~S}$ ribosomal copy number. Interestingly, eight of the compounds $(1,3,5,19,21,29,32$, and 36 ) produced a trend towards increased infectivity of sporozoites.

\section{Initial cytotoxicity study on human hepatocytes via flow cytometry}

Those compounds showing activity in vitro and in vitro parasite assays (Fig. 5; Additional file 6) were also assayed for their effects on the viability of human HC-04 liver cells. As shown in Fig. 6, preliminary studies using $1 \mathrm{mM}$ of five selected compounds in $1 \%$ final DMSO concentration do not show the induction of apoptotic markers as assayed by annexin $\mathrm{V}$ and phosphoinositol (PI)-staining after $96 \mathrm{~h}$ incubation. Taking these results together, they suggest that these compounds are indeed parasite-specific.

\section{Ternary co-crystal structure confirms mode of action of compound 24}

Well-diffracting crystals of the ternary complex PfAldolase-TRAP were obtained in the presence of various compounds. However in only one case, that of compound 24 , clear electron density was observed for both TRAP and the compound in all four sub-units of the aldolase tetramer (Fig. 7a; Additional files 7, 8, 9, 10). Notably, in the previously published aldolase-TRAP co-crystal structure (PDB ID: 2pc4) [26], TRAP could only be seen in one out of four aldolase sub-units, further highlighting the stabilizing effect of this compound on the complex. It is worth noting that the superposition comparison between 2pc4 [26] and the aldolase-TRAP-compound 24 ternary complex reveals significant shifts of the TRAP-binding position upon addition of compound 24 . The W604 ring system varies only slightly in its position by $0.8 \AA$ (Fig. 7b), whereas the D603 $\mathrm{C}_{\mathrm{a}}$-position shifts by $3.6 \AA$ and the N605 $\mathrm{C}_{\mathrm{a}}$-position by $4.7 \AA$ (Fig. 7c). The largest observed distance between the two crystal structures is the side chain of residue D603 with $8 \AA$. Overall, the TRAP-tails are pushed further into the pocket of aldolase upon addition of the small molecule stabilizer, compound 24, thereby occluding the F16P substratebinding site (Additional file 11). The dihydroxybenzylring of compound 24 stays within the experimental error of the X-ray crystal structure in the same position in all four sub-units, contacting residue N606 of TRAP and N39, E40, T44 of aldolase through hydrophobic interactions. The dichlorobenzyl-ring system adopts two major conformations, indicating flexibility of the compound when bound to the PfAldolase-TRAP interface (Figs. 7c, 8 , Additional files $3,4,5,6,7,8,9,10)$. The contacting residues on aldolase mainly provide hydrophobic interactions mediated through residues L117, R158 and L198 for binding mode 1 and T44, K47 and R48 in the alternative conformation, while also contacting D604 of the TRAPtail. A future derivative of compound 24 with a double ring system at this position may confer higher binding 
a
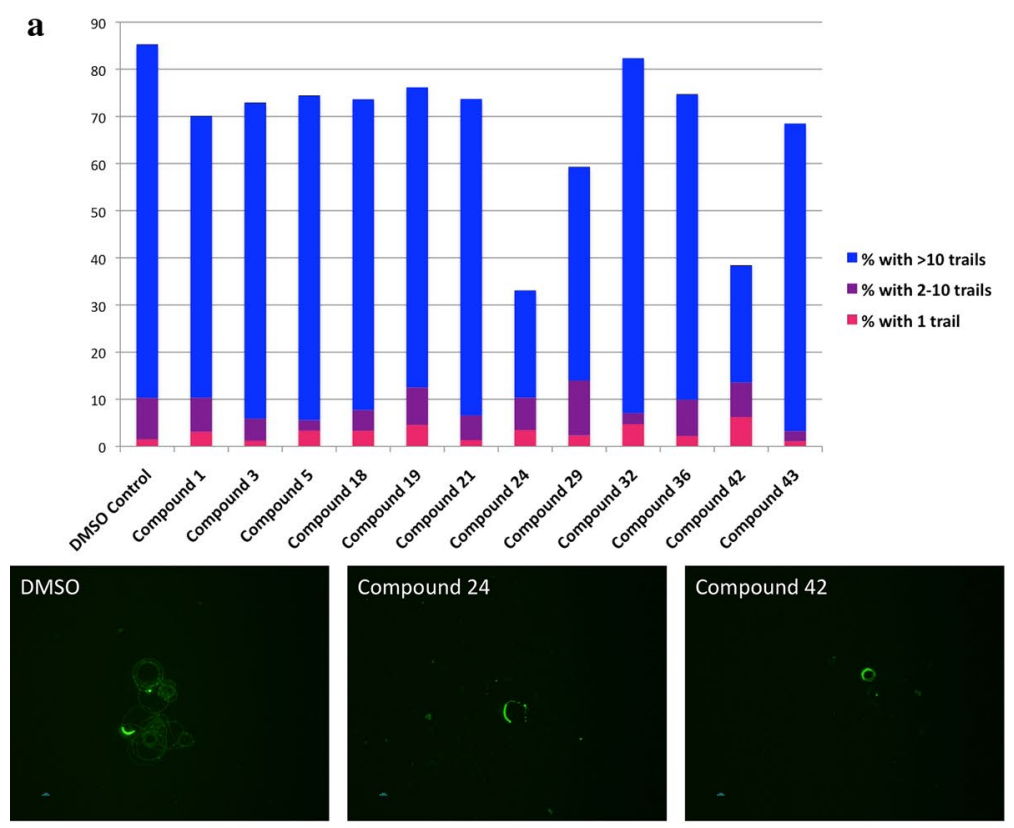

b

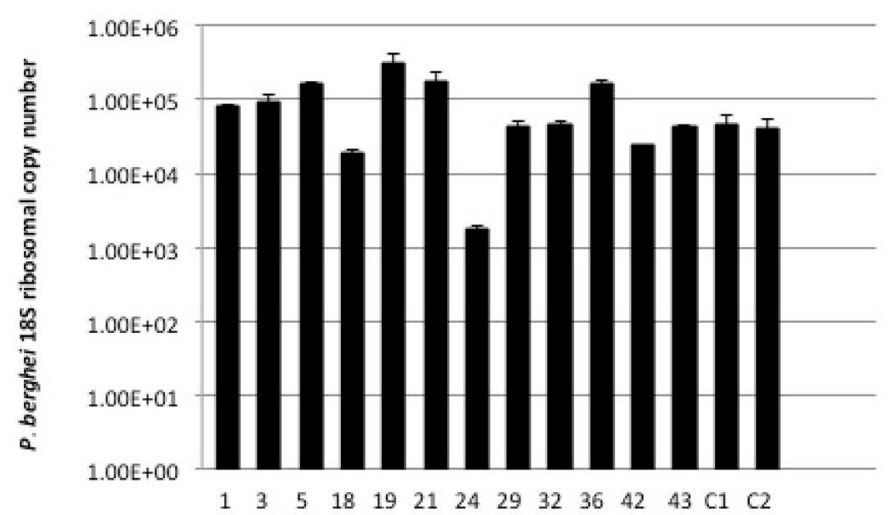

Compound \#

c

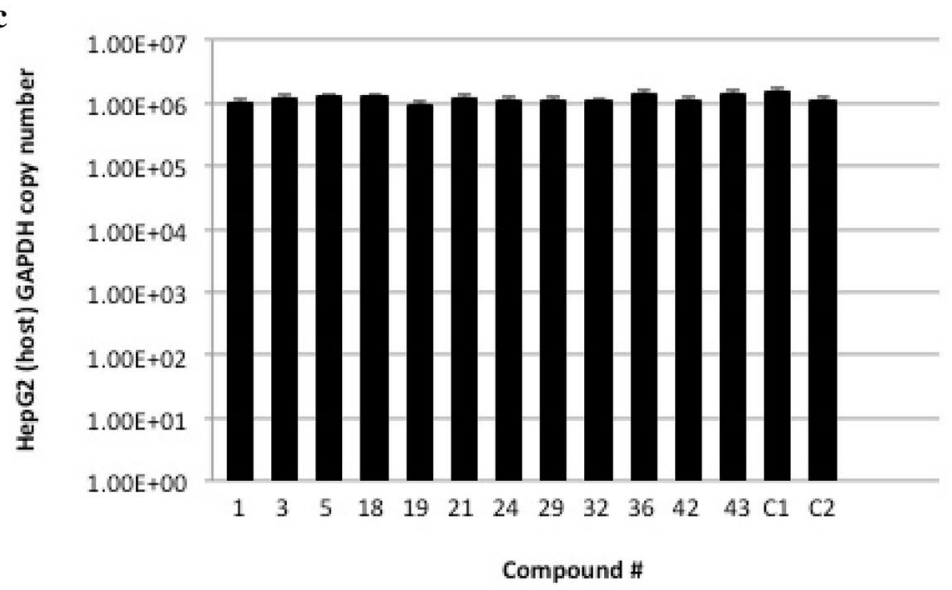


(See figure on previous page.)

Fig. 5 Gliding motility and hepatocyte invasion assay in the presence of compounds. a Compounds 24 and 42 impair the ability of $P$. berghei sporozoites to glide on glass coverslips. The total height of bars represent the percentage of sporozoites that were motile (produced one or more trails on a glass slide) during the assay period. Untreated sporozoites produce $>10$ trails under the assay conditions. As shown here, sporozoites treated with compounds 24 or 42 were less motile overall and produced fewer trails than the DMSO controls. In this experiment, treatment with DMSO, compound 24 , or compound 42 produced 85,33 , and $38 \%$ motile sporozoites, respectively. Fluorescent microscopy images of a representative sporozoite (green crescent) and its gliding path (green spirals) for three assay treatments: DMSO control (left), compound 24 (centre), and compound 42 (right). Sporozoite trails were visualized using a biotinylated antibody to CSP. While some sporozoites treated with compounds 24 or 42 did produce $>10$ trails, most of them produced no or few trails as shown here. $\mathbf{b}$ HepG2 liver invasion assay in the presence of small molecules. Compounds 18 , 24 , and 42 produced 58.9, 95.6 and $34 \%$ inhibition of invasion, respectively. Note the logarithmic scale. c Real time PCR of the host cell GAPDH mRNA was used as a control for equal amounts present in each assay.

affinities to the PfAldolase-TRAP complex by decreasing the rotational freedom of the compound in the binding site.

When comparing the predicted binding pose of compound 24 from VLS with the actual experimentally observed in the crystal structure, one can observe a reasonable good agreement of the proximity of the ligand (Additional file 12). The motion of the TRAP-peptide deeper into the PfAldolase active site introduces significant changes that currently cannot be computationally predicted a priori. When re-docking compound 24 to the actual observed ternary co-crystal structure (4TR9), the

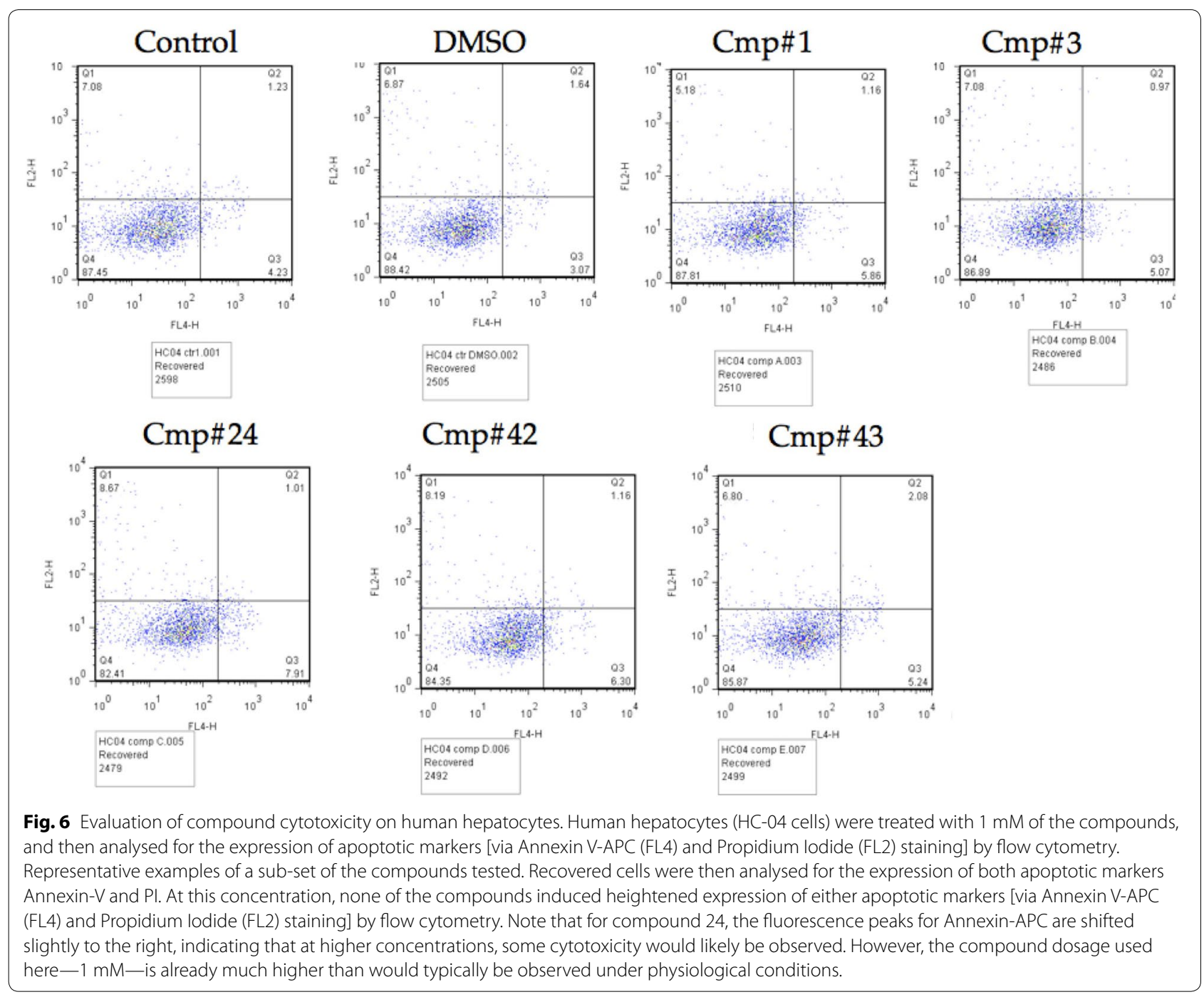




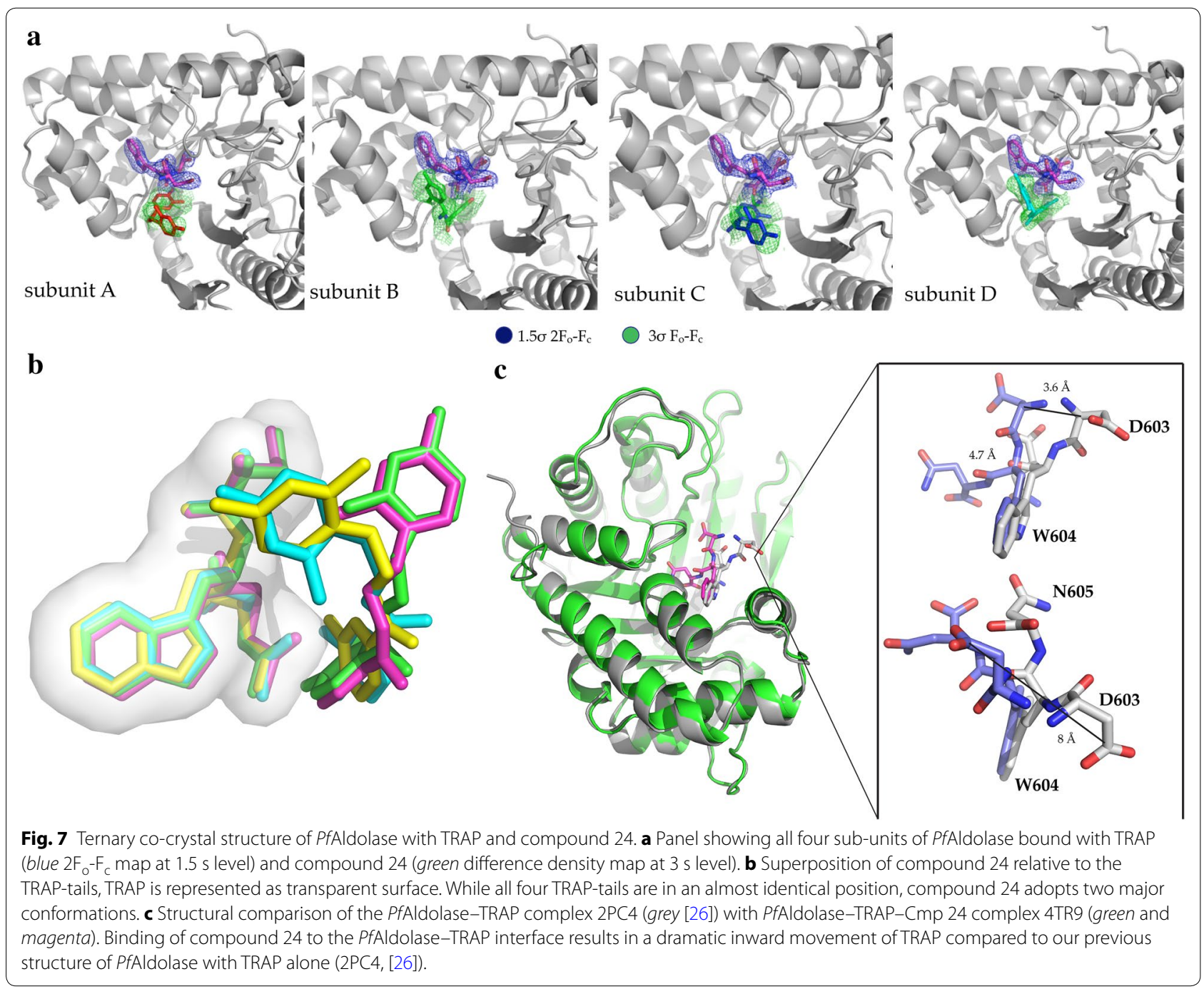

predicted pose results in a better overlap, with a preference for the binding mode 2 where the dichlorobenzyl-ring system is in contact with T44, K47 and R48 (Additional file 12).

\section{Compound 24 does not cross-react with rabbit aldolase}

To validate that this initial hit compound represents a viable drug candidate, enzymatic assays were performed with rabbit aldolase, which is $99 \%$ sequence identical to human aldolase (Additional file 1). Only four residues out of 364 vary between these two species, while Plasmodium shares only $56 \%$ sequence identity (203/364 identical residues) with rabbit aldolase or human aldolase (Additional file 1). A dilution series of compound 24, ranging from 250 to $0 \mu \mathrm{M}$ was tested with rabbit aldolase, PfAldolase, and PfAldolase in the presence of the TRAPpeptide. While compound 24 did not induce a significant change in activity in the rabbit aldolase or PfAldolase alone, a dramatic change in activity was observed upon addition of both the TRAP-peptide and compound 24 to PfAldolase, further supporting the initial hypothesis of compound 24 acting as a stabilizing agent of the PfAldolase-TRAP protein-protein interaction (Fig. 9). Compound 24 inhibits PfAldolase catalytic activity only in the presence of TRAP.

\section{Surface plasmon resonance (SPR) studies indicate decreased dissociation rates of the PfAldolase-TRAP complex in the presence of compound 24}

The authors recently devised a method [34] by which screening and characterization of small molecules that enhance the binding of PfAldolase and TRAP can be performed. The binding of either PfAldolase to immobilized TRAP peptides or binding of TRAP to immobilized 

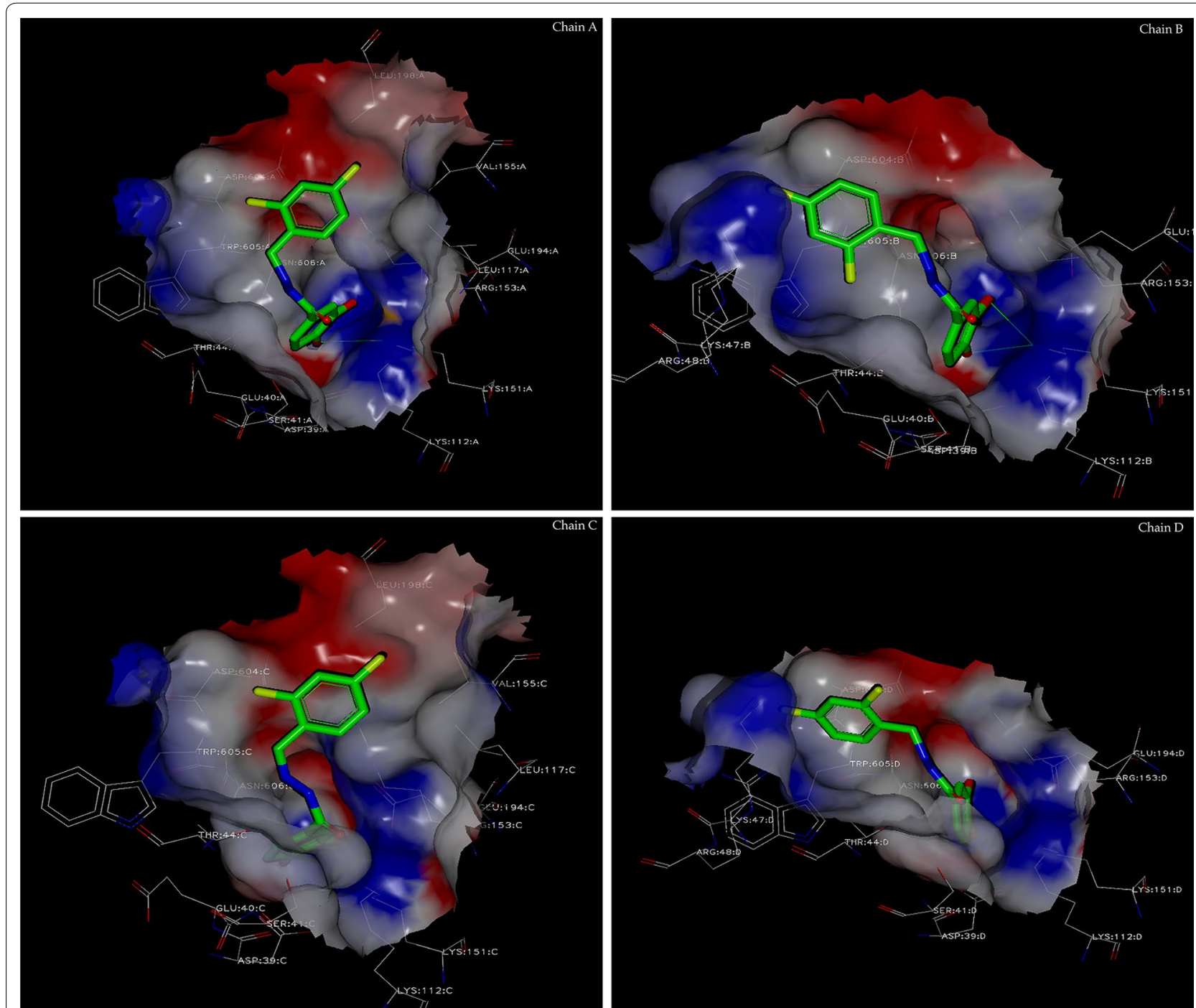

Fig. 8 Detailed interaction representation of compound 24 with the PfAldolase-TRAP interface. The electrostatic surface of the mixed interface is shown and compound 24 is kept in a green-stick representation. Figure generated in Vida [71] and rendered with Povray [72].

PfAldolase on a SPR chip has been previously demonstrated [34, 45]. In this study PfAldolase was passed over a chip with immobilized TRAP-peptides to measure the binding and dissociation in the presence of compound 24 (Fig. 10). The association constant $\left(\mathrm{K}_{\mathrm{a}}\right)$ decreases and the dissociation rate $\left(\mathrm{K}_{\mathrm{d}}\right)$ increases in the presence of compound 24 , thereby indicating a stabilizing effect on the PfAldolase-TRAP complex under physiological buffer conditions. A dose-dependent delay in dissociation of $\sim 5 \mathrm{~s}$ can be observed with concentrations $>500 \mu \mathrm{M}$ as indicated by dotted lines in Fig. 10. At the highest concentration tested of $1,000 \mu \mathrm{M}$ the dissociation is delayed by $\sim 10 \mathrm{~s}$, providing a biophysical real time observation of the binding promoting capabilities of compound 24 . No delayed dissociation effect is observed with lower concentrations of the compound than those depicted.

\section{Discussion}

Here, the challenge to pharmacologic targeting of a site strongly conserved in amino acid sequence between human and parasite in the key aldolase protein of the Plasmodium glideosome was overcome by successfully designing a screen for compounds that specifically stabilize a key protein-protein interface between PfAldolase and TRAP in the malaria parasite. The proposed binding mode of the stabilizing compound was confirmed in the ternary Aldolase-TRAP-C24 co-crystal structure (Figs. 7, 8, Additional files 7, 8, 9). Since the compounds 


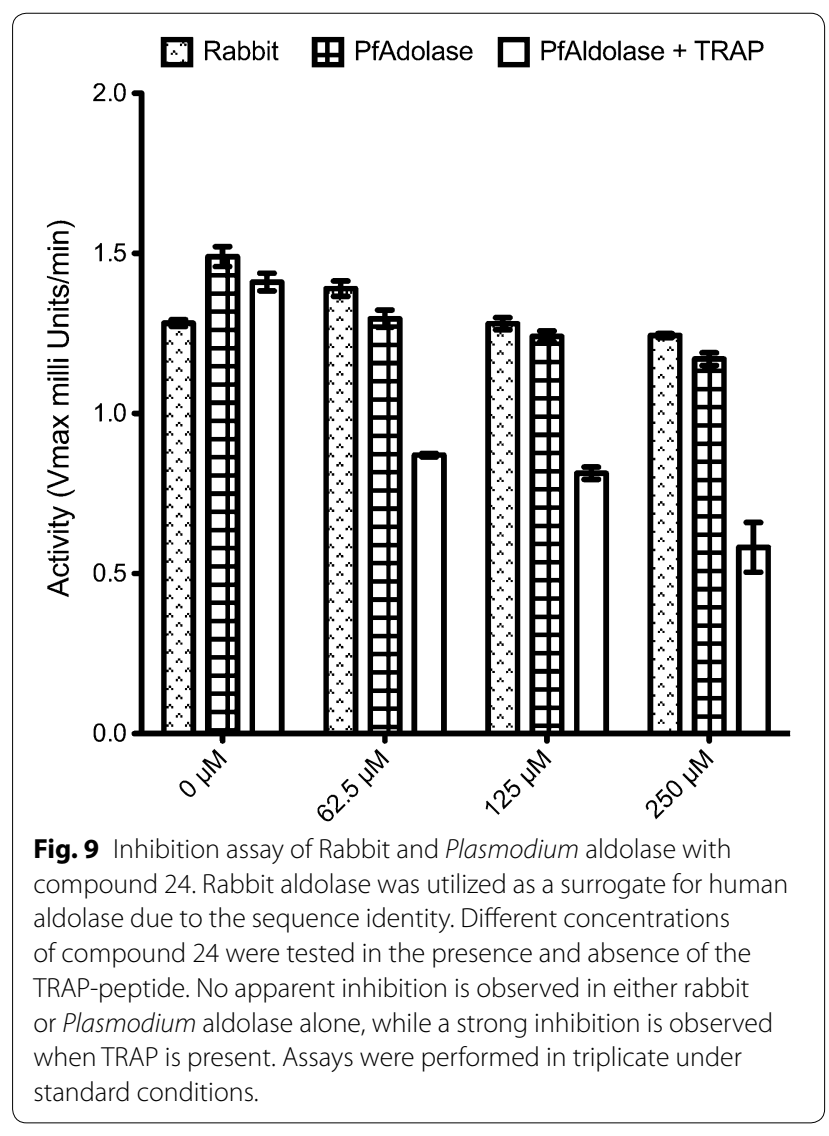

also inhibited parasite motility and invasion (Figs. 5, 11, Additional file 6), they suggest that this interface in the glideosome is a relevant target for anti-malarial drug design. Furthermore, an additional effect of stabilizing the PfAldolase-TRAP interface may result in the occlusion of the actin-binding site to PfAldolase and thereby preventing actin binding, which is needed for a productive invasion event, hence potentiating the effect of the stabilizing ligand. Importantly, by stabilizing TRAP bound to aldolase, compound 24 also potentiates aldolase inhibition by the TRAP protein (Figs. 7, 8, Additional file 9), enhancing their appeal as drug candidates. A ternary complex of aldolase-TRAP and compound 24 can be rendered catalytically inactive with appropriate compound concentrations, thereby having a limiting effect on energy production of the parasite through glycolysis. Further structural and biochemical studies should help delineate the extent to which inhibition of glycolysis contributes to the compounds' anti-infective effect versus direct interference with the glideosome's physical mechanism of movement generation.

Of note, recent studies have called into question the conservation of the gliding machinery across Apicomplexa, and in particular the role played by aldolase in

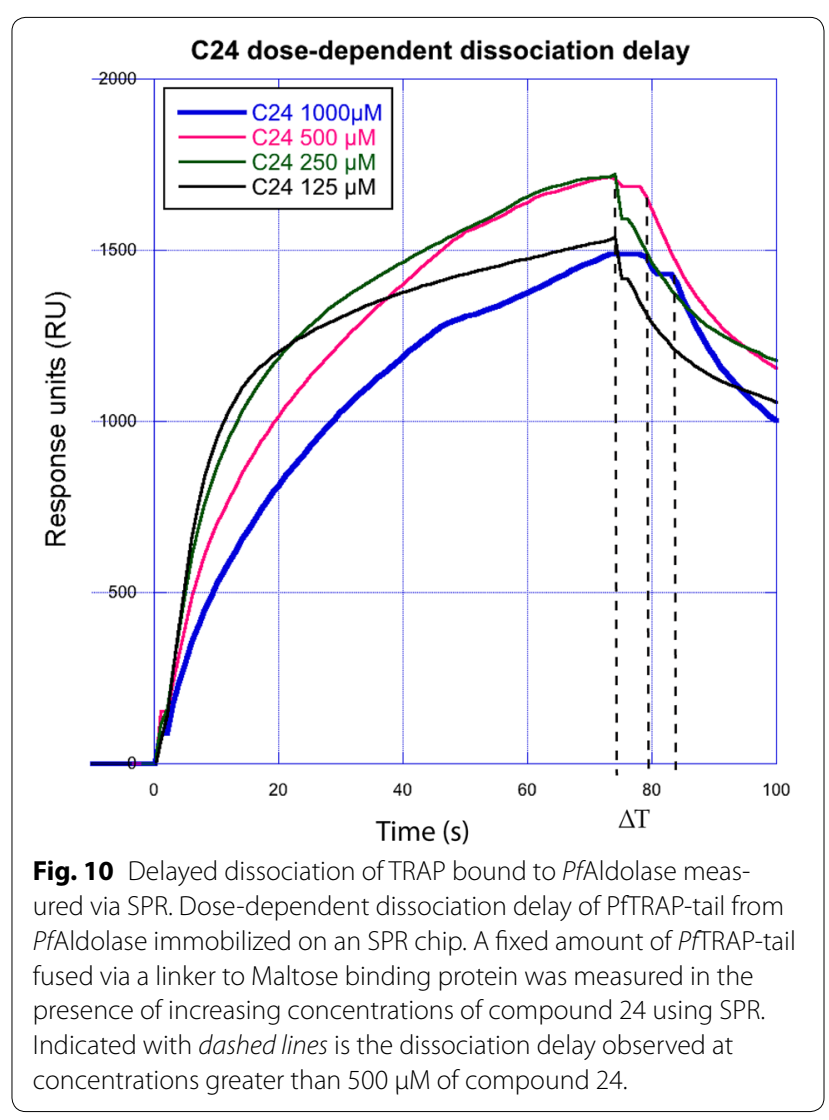

infectivity. Shen et al. showed that one aldolase isozyme (TgALD1) can be knocked out, and the altered parasites retain infectivity, although to a much lower degree [46]. In contrast, a recent study utilizing biolayer interferometry, as well as co-sedimentation studies, confirmed that aldolase and actin are required for Plasmodium for cell invasion of host cells [47], although this individual study is not considered definitive. The reader is referred to a recent comprehensive review discussing the similarities and differences of the glideosomes in Toxoplasma and Plasmodium [48] In addition to representing potential new leads in anti-malarial drug design, the compounds and methods described here represent an important new strategy for the field by providing new pharmacology (non-genetic) tools that may help clarify the Toxoplasma genetic studies. The most selective compounds could be valuable non-genetic tools for further investigating glideosome function in Plasmodium. At the concentrations tested, compound 24 did not inhibit rabbit aldolase, which was utilized as a surrogate for human aldolase with $99.3 \%$ sequence identity (Fig. 9; Additional file 1). In addition, our studies suggest that the genetic findings in Toxoplasma may not apply to other biomedically important members of the Apicomplexan phylum [26]. 


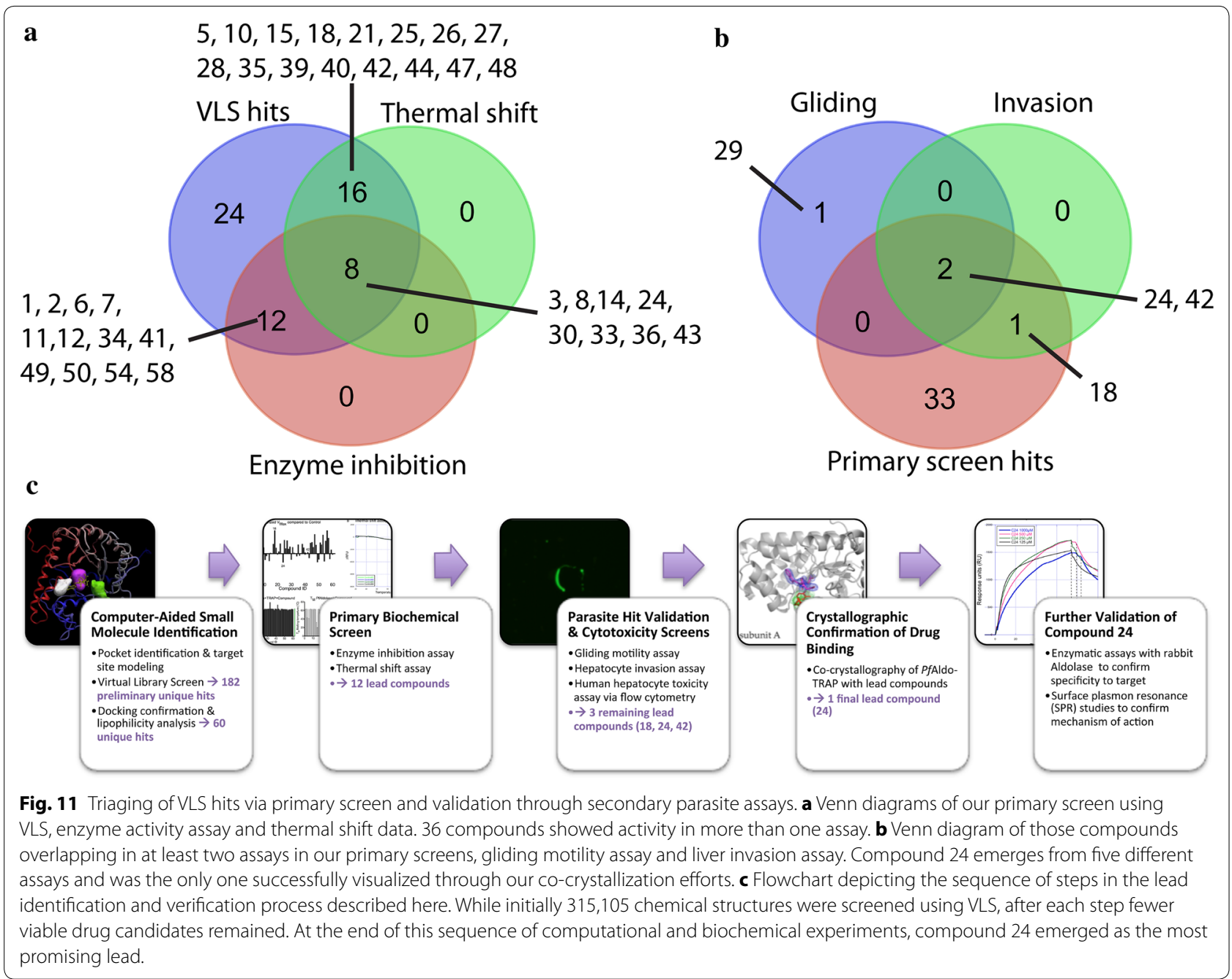

Although proof-of-principle was achieved, the compounds exhibit relatively low potencies in functional assays, which is a limitation for their development into drug leads. Nevertheless, the potency of the hits themselves may be less important than validation of the target drug-binding pocket by structure-based screening: the pharmacophore space that is now mapped out by the hits and their bioactivities, in conjunction with the known interactions within the X-ray crystal structure, can serve as a blueprint for rational optimization of the hits in multiple directions (better potency, better bioavailability, etc.), using resources such as medicinal chemistry and fragment-based tethering. Notably, the GSK TCAMS [49] Novartis-GNF Malaria Box, and St Jude Children's Research Hospital [50] datasets of hits from whole-cell screenings against $P$. falciparum blood stages include several compounds containing the $\mathrm{N}$-(benzylideneamino) benzamide scaffold between them (Additional file 13). This scaffold is shared by some of the hits identified here
(Fig. 4a), and medicinal chemistry derivatization of this scaffold may yield additional compounds with greater potency against the parasite or other favourable drug properties. It should also be noted that the average concentration of FDA approved drugs used to treat malarial disease in humans is close to $500 \mu \mathrm{M}$. For example, when injected intravenously the usual formulation of Chloroquine employed is $200 \mathrm{mg} / \mathrm{ml}$, resulting in approximately $100 \mu \mathrm{M}$ final concentration in the blood. Malarone, a combination therapy of atovaquone and proguanil is given as an oral dose at $750 \mathrm{mg} / 5 \mathrm{ml}(\sim 410 \mathrm{mM})$, corresponding to approximately $30 \mu \mathrm{M}$ final concentration for a $60-\mathrm{kg}$ person, assuming equal biodistribution throughout the body [51].

One intuitive, theoretical concern related to this study is that compounds targeted to the PfAldolase-TRAP interface may actually destabilize the complex or that stabilizing the target interface might enhance motility and infectivity. Indeed, several compounds showed destabilization on 
thermal shift. Notably, only the last three residues of TRAP (604-605) were visible in the crystal structure and receptor models which were screened against here. It is possible that these compounds interact in an inhibitory way with upstream TRAP residues that could not be accounted for in this screen. If so, these compounds may be the basis for a new approach to inhibit the glideosome as they should be even more specific. Destabilizing compounds, although not desired by the present design, may actually be useful from a drug development point of view once their exact mechanism of action is known. Additionally, eight compounds showed insignificant trends towards increases in invasion despite no change or decreases in motility, possibly indicating that some of the compounds may have additional off-target effects or change the PfAldolase-TRAP interaction in a way that increases invasion but not motility. As the precise chemistry and mechanism of the glideosome is still obscure, these possibilities cannot be ruled out. Compounds having this effect may nevertheless be useful non-genetic tools for studying precise glideosome sub-mechanisms.

Most drugs in use today inhibit biological interactions. However, the scientific literature contains several examples of biologically active small molecules that function by stabilizing protein-protein interactions in a bipartite manner, including fungal toxins [52-54], chemotherapeutic agents [55-59], antibiotics [60,61], and immunosuppressants [62]. These examples indicate, and the results here ultimately suggest, that it could be possible to develop clinically useful compounds that enhance PPI. For a small molecule to inhibit a PPI, it must bind to its receptor with a higher affinity than, and at least similar specificity to, the protein's native ligand. A vast collection of failed drug candidates demonstrates how difficult it is to compete with eons of evolutionary pressure that produced the biomolecular interaction in the first place [63]. Stabilizing that interaction, however, does not require competing with nature, as a nearby region is targeted by the small molecule. Rather, this approach tries to nudge the interaction's equilibrium in the direction that is thermodynamically favoured to begin with. Thus a candidate enhancer does not need to bind either member of a protein complex with particularly high affinity-it is the aggregate of affinities of the proteins for each other and for the drug that matter [54]. As demonstrated by the compounds discovered here, adding just one or two contact points to a protein complex can make a very big difference in its stability.

The enhancer approach may work especially well for situations in which the conformational dynamics of a protein complex are key to its function. In this case, the ability of the glideosome to provide the motive force is dependent on the highly coordinated interactions of its members. Aldolase must tightly bind both actin and TRAP to allow motion to begin, but it must also rapidly release the TRAP tail after its cleavage to allow motion to continue. While it is unclear if the same pool of aldolase participates in both motility and glycolysis, the enzymatic binding and cleavage of F16P is crucial for providing the ATP molecules necessary for the actin-myosin power stroke [29, 64]. The various conformations of aldolase, TRAP, actin, and MyosinA must therefore exist in the ideal equilibriums to promote the proper bind-andrelease sequences for each of the glideosome interactions. Shifting these equilibrium in either direction by inhibiting or enhancing any of the interactions involved should affect the motor. The computer-aided, structurebased approach to drug discovery presented here allowed the specific targeting of structural differences between multiple conformations of aldolase in order to shift the apo-aldolase/aldolase-F16P/aldolase-TRAP equilibrium towards the aldolase-TRAP complex in a parasiteselective fashion. Nature abounds with similar vulnerable systems of exquisitely regulated biological motors and complexes, many of which might be targeted by this structure-based enhancer method.

Future modelling and crystallographic studies should help define additional receptor pockets and conformations that can be exploited to design compounds that target different aspects of the glideosome, including the glideosome homologs present in different stages of the Plasmodium life-cycle, as well as glideosome components conserved in other apicomplexan pathogens, such as Toxoplasma gondii and Cryptosporidium spp. For example, given sufficient structural information, the interactions between MTIP and Myosin A in Plasmodium could also be targeted in a similar fashion to the TRAP-aldolase complex by either stabilizing the close conformation or preventing opening of the EF-hands of MTIP. Several structures of PfMTIP and PkMTIP in complex with Myo$\sin$ A [65-67] and stapled peptides [68, 69] have been described to date. Analysis of their structural flexibility may provide crucial insights towards targetable interfaces and pockets.

While stabilizing the PfAldolase-TRAP interaction may seem like an unusual approach for rational drug design, by targeting this joint surface, the avenue it opens to promoting parasite specificity-TRAP is not present in humans-may prevent the emergence of resistance. Additionally, resistance mutations in aldolase's active site would be highly unfavourable as they would likely interfere with glycolytic energy generation. Starnes et al. [64] demonstrated in Toxoplasma that mutations near the active site of aldolase are not tolerated by the parasite, which is in agreement with the decreased likelihood of resistance mutations emerging. 
The strategy of targeting a hybrid surface composed of a conserved target and a non-conserved target for the purposes of combating resistance may be broadly applicable to structure-based drug design. This may be especially useful for developing agents to fight eukaryotic pathogens, as many of their essential proteins have highly conserved human homologues, and are otherwise difficult to specifically target. It may also be possible to use this type of approach to design therapeutics for rapidly mutating viruses by selectively modulating host-pathogen interactions, i.e. preventing the dissociation of a viral surface protein with its host receptor may increase the virus's vulnerability to other drugs or to the host's own immune system. In this case, the druggable surface encompassing the viral protein would provide specificity, while the unlikelihood of mutations in the host protein may protect against the development of drug resistance. Similarly, one can envision targeting a complex of a normal housekeeping protein and a mutant oncoprotein to selectively kill cancer cells.

It is important to note that the compounds and chemical scaffolds identified here are not found among the anti-malarials currently in clinical use. The fact, that the identified compound emerged directly from a VLS effort and has not undergone any chemical optimization, while showing an on-target effect by various biophysical and parasitological assays bodes well for its future development from a probe to a lead compound. This effort, therefore, represents a successful 'scaffold hop' in antimalarial drug discovery. If ultimately successful, these drugs and their derivatives would constitute a novel class or classes of anti-malarial agents, as well as the first drugs to target the aldolase-TRAP interaction.

At present, a more potent, safe drug with an identical mechanism of action to compound 24 could be useful for malaria prophylaxis, but its activity against merozoites, the extracellular form of the erythrocytic stage parasites was negative, likely due to the additional C-terminal extension of the merozoite TRAP homologue MTRAP occluding binding of compound 24. Nevertheless, if the compounds identified here do not cross-react with merozoites, a similar screen to that described in this study, targeting MTRAP, the merozoite homologue of TRAP, could yield a similar, specific drug active in the blood stages. Based on the sequence identity of the terminal residues of TRAP with CTRP, the circumsporozoite TRAP-like protein (PlasmoDB code PF3D7_0315200) expressed during the mosquito stage, it is anticipated that compound 24 may function as a stabilizer of the PfAldolase-CTRP interface and be able to block mosquito midgut invasion of the parasite. Such a compound could perhaps be used as a spray to treat bednets or to treat hatching areas and prevent spread of mosquitoes carrying the parasite.

\section{Conclusion}

In summary, the results presented here validate the aldolase-TRAP interaction within the Plasmodium glideosome as a drug discovery target, by proving both that it can be pharmacologically targeted and that doing so does affect the parasite's motility and invasion capabilities. It remains to be determined, if a similar approach can succeed in Toxoplasma and other Apicomplexa, but using the chemical probes discovered here may contribute to the understanding of the role of aldolase in Toxoplasma gliding motility.

This work also provides proof-of-concept that the structure-based, selective, enhancement of PPI is a viable, efficient and effective method of novel drug hit discovery, opening new avenues to drug discovery for challenging targets, such as the glideosome.

\section{Additional files}

Additional file 1. Clustal-W sequence alignment of Plasmodium, human and rabbit aldolase.

Additional file 2. Pockets in the unliganded aldolase crystal structure.

Additional file 3. Receptor models for VLS and docking boxes.

Additional file 4. VLS hit lists and compound structures and properties.

Additional file 5. Stereo figures of selected VLS hit poses.

Additional file 6. Sporozoite motility assay results for all compounds tested.

Additional file 7. Ligplot representation of the TRAP peptides.

Additional file 8. Ligplot representation of the Compound 24 molecules.

Additional file 9. Stereo figures of each PfAldolase subunit bound to TRAP and compound 24.

Additional file 10. Overview of the PfAldolase ternary complex structure. Individual PfAldolase subunits are colored by chain. The TRAP-peptide present in all four subunits is shown as stick representation as well as Compound 24. The movie zooms onto each sub-unit and represents the individual binding sites. At the end a sphere model of TRAP and Compound 24 is shown to illustrate possible areas, which could be filled during future SAR studies.

Additional file 11. Superposition of Plasmodium aldolases apo, TRAPbound and compound 24 and TRAP-bound form. Colour coding of the structure is kept identical to Fig. 5C.

Additional file 12. Stereo figure of compound 24 in the crystal structure compared to the docking pose obtained with 2PC4.

Additional file 13. Identified C24 scaffold homologs within the TCAMs dataset.

\section{Authors' contributions}

The enhancer approach was initially conceived by SMN and TC. All computational work was performed by SMN under the guidance of TC. Aldolase plasmids were generated by $C B, G B$ and JB. Synthetic peptides were designed by SMN, GB, JB, TC, CB, and VN. Catalysis assays were performed by SMN under the guidance of TC, and by KO and RW under the guidance of JB. Thermal shift assays and X-ray crystallography were performed by GB, KO and RW under the guidance of JB. Sporozoite motility assays were performed by IE under the guidance of PS. Invasion assays were performed by KAK under the guidance of VN. Hepatocyte toxicity assays were performed by JL. Surface plasmon resonance assays were performed by JB. All authors contributed to the writing and 
editing of the manuscript, as well as to scientific discussions and experimental design. All authors read and approved the final manuscript.

\begin{abstract}
Author details
1 Present Address: Department of Biochemistry and Molecular Pharmacology, New York University School of Medicine, New York, USA. ${ }^{2}$ Institute for Systems Genetics, New York University School of Medicine, New York, USA. ${ }^{3}$ Department of Molecular Microbiology and Immunology, Johns Hopkins University Bloomberg School of Public Health, Baltimore, USA. ${ }^{4}$ Department of Biochemistry and Molecular Biology, Johns Hopkins University Bloomberg School of Public Health, Baltimore, USA. ${ }^{5}$ Department of Medical Parasitology, New York University School of Medicine, New York, USA. ${ }^{6}$ Michael Heidelberg Division of Pathology of Infectious Diseases, Department of Pathology, New York University School of Medicine, New York, USA. ${ }^{7}$ Instituto de Investigaciones Biotecnológicas-Instituto Tecnológico de Chascomús (IIB-INTECH), Universidad Nacional de General San Martín-CONICET, 1650 San Martín, Buenos Aires, Argentina. ${ }^{8}$ Johns Hopkins Malaria Research Institute (JHMRI), Baltimore, USA. ${ }^{9}$ Present Address: Department of Pediatrics, Phyllis and David Komansky Center for Children's Health, New York-Presbyterian Hospital-Weill Cornell Medical College, New York, USA. ${ }^{10}$ Present Address: Department of Animal Sciences, School of Life Sciences, University of Hyderabad, Hyderabad 500046, India.
\end{abstract}

\section{Acknowledgements}

We thank Keren Klein, Lily Wu, Sandra Knoll, Dafna Gershoony, and Meredith Barton for technical assistance with experimental protocols. Lily Wu is also acknowledged for her helpful comments on the manuscript. We particularly thank Pete Dunten, Irimpan Mathews and the staff at SSRL beamline 9-2 and beamline 12-2, as well as Peter Zwart and the staff at ALS beamline 5.0.1 for their help and assistance during synchrotron data collection.

\section{Protein data base (PDB) accession code}

Coordinates and structure factors have been deposited with the PDB under accession code 4TR9.

\section{Funding}

This work was funded by NIH Grants DP2 OD004631 to TC, F30HL094052 to SMN, 5R01Al056840 to PS and a minority supplement to this grant to IE and by funding from the NYU MSTP program to SMN and IE. This work was partially funded through The Bloomberg Family Foundation (GB, RW, PS, JB), and a pilot Grant from the Johns Hopkins Malaria Research Institute (JL, JB).

\section{Compliance with ethical guidelines}

\section{Competing interests}

A patent application based on the presented results has been submitted by TC, JB and SMN.

Received: 11 June 2015 Accepted: 2 August 2015

Published online: 20 August 2015

\section{References}

1. Baird JK (2005) Effectiveness of antimalarial drugs. N Engl J Med 352:1565-1577. doi:10.1056/NEJMra043207

2. Jambou R, Legrand E, Niang M, Khim N, Lim P, Volney B et al (2005) Resistance of Plasmodium falciparum field isolates to in vitro artemether and point mutations of the SERCA-type PfATPase6. Lancet 366:1960-1963. doi:10.1016/S0140-6736(05)67787-2

3. Phyo AP, Nkhoma S, Stepniewska K, Ashley EA, Nair S, McGready R et al (2012) Emergence of artemisinin-resistant malaria on the western border of Thailand: a longitudinal study. Lancet 379:1960-1966. doi:10.1016/ S0140-6736(12)60484-X

4. Saralamba S, Pan-Ngum W, Maude RJ, Lee SJ, Tarning J, Lindegardh N et al (2010) Intrahost modeling of artemisinin resistance in Plasmodium falciparum. Proc Natl Acad Sci USA 108:397-402. doi:10.1073/ pnas. 1006113108

5. Ariey F, Witkowski B, Amaratunga C, Beghain J, Langlois AC, Khim N et al (2014) A molecular marker of artemisinin-resistant Plasmodium falciparum malaria. Nature 505:50-55. doi:10.1038/nature12876
6. Baum J, Papenfuss AT, Baum B, Speed TP, Cowman AF (2006) Regulation of apicomplexan actin-based motility. Nat Rev Microbiol 4:621-628

7. Sibley DL (2010) How apicomplexan parasites move in and out of cells. Curr Opin Biotechnol 21:592-598

8. Montagna GN, Matuschewski K, Buscaglia CA (2012) Plasmodium sporozoite motility: an update. Front Biosci 17:726-744

9. Collingridge PW, Brown RWB, Ginger ML (2010) Moonlighting enzymes in parasitic protozoa. Parasitology 137:1467-1475. doi:10.1017/ S0031182010000259

10. Bergman LW, Kaiser K, Fujioka H, Coppens I, Daly TM, Fox S et al (2003) Myosin A tail domain interacting protein (MTIP) localizes to the inner membrane complex of Plasmodium sporozoites. J Cell Sci 116:39-49

11. Bartholdson SJ, Crosnier C, Bustamante LY, Rayner JC, Wright GJ (2013) Identifying novel Plasmodium falciparum erythrocyte invasion receptors using systematic extracellular protein interaction screens. Cell Microbiol 15:1304-1312. doi:10.1111/cmi.12151

12. Heiss K, Nie H, Kumar S, Daly TM, Bergman LW, Matuschewski K (2008) Functional characterization of a redundant Plasmodium TRAP family invasin, TRAP-like protein, by aldolase binding and a genetic complementation test. Eukaryot Cell 7:1062-1070. doi:10.1128/EC.00089-08

13. Moreira CK, Templeton TJ, Lavazec C, Hayward RE, Hobbs CV, Kroeze $\mathrm{H}$ et al (2008) The Plasmodium TRAP/MIC2 family member, TRAP-like protein (TLP), is involved in tissue traversal by sporozoites. Cell Microbiol 10:1505-1516. doi:10.1111/j.1462-5822.2008.01143.x

14. Müller HM, Reckmann I, Hollingdale MR, Bujard H, Robson KJ, Crisanti A (1993) Thrombospondin related anonymous protein (TRAP) of Plasmodium falciparum binds specifically to sulfated glycoconjugates and to HepG2 hepatoma cells suggesting a role for this molecule in sporozoite invasion of hepatocytes. EMBO J 12:2881-2889

15. Siddiqui FA, Dhawan S, Singh S, Singh B, Gupta P, Pandey A et al (2013) A thrombospondin structural repeat containing rhoptry protein from Plasmodium falciparum mediates erythrocyte invasion. Cell Microbiol 15:1341-1356. doi:10.1111/cmi.12118

16. Srinivasan $P$, Beatty WL, Diouf A, Herrera R, Ambroggio X, Moch JK et al (2011) Binding of Plasmodium merozoite proteins RON2 and AMA1 triggers commitment to invasion. Proc Natl Acad Sci USA 108:13275-13280. doi:10.1073/pnas.1110303108

17. Baker RP, Wijetilaka R, Urban S (2006) Two Plasmodium rhomboid proteases preferentially cleave different adhesins implicated in all invasive stages of malaria. PLoS Pathog 2:e113. doi:10.1371/journal.ppat.0020113

18. Ejigiri I, Ragheb DRT, Pino P, Coppi A, Bennett BL, Soldati-Favre D et al (2012) Shedding of TRAP by a rhomboid protease from the malaria sporozoite surface is essential for gliding motility and sporozoite infectivity. PLoS Pathog 8:e1002725. doi:10.1371/journal.ppat.1002725

19. Kappe SHI, Buscaglia CA, Nussenzweig V (2004) Plasmodium sporozoite molecular cell biology. Annu Rev Cell Dev Biol 20:29-59. doi:10.1146/ annurev.cellbio.20.011603.150935

20. Bargieri D, Lagal V, Tardieux I, Ménard R (2012) Host cell invasion by apicomplexans: what do we know? Trends Parasitol 28:131-135. doi:10.1016/j.pt.2012.01.005

21. Thiel P, Kaiser M, Ottmann C (2012) Small-molecule stabilization of protein-protein interactions: an underestimated concept in drug discovery? Angew Chem Int Ed Engl 51:2012-2018. doi:10.1002/anie.201107616

22. Döbeli H, Trzeciak A, Gillessen D, Matile H, Srivastava IK, Perrin LH et al (1990) Expression, purification, biochemical characterization and inhibition of recombinant Plasmodium falciparum aldolase. Mol Biochem Parasitol 41:259-268

23. Mecozzi VJ, Berman DE, Simoes S, Vetanovetz C, Awal MR, Patel VM et al (2014) Pharmacological chaperones stabilize retromer to limit APP processing. Nat Chem Biol 10:443-449. doi:10.1038/nchembio.1508

24. Schapira M, Raaka BM, Das S, Fan L, Totrov M, Zhou Z et al (2003) Discovery of diverse thyroid hormone receptor antagonists by high-throughput docking. Proc Natl Acad Sci USA 100:7354-7359

25. An J, Totrov M, Abagyan R (2005) Pocketome via comprehensive identification and classification of ligand binding envelopes. Mol Cell Proteom 4:752-761. doi:10.1074/mcp.M400159-MCP200

26. Bosch J, Buscaglia CA, Krumm B, Ingason BP, Lucas R, Roach C et al (2007) Aldolase provides an unusual binding site for thrombospondin-related anonymous protein in the invasion machinery of the malaria parasite. Proc Natl Acad Sci USA 104:7015-7020. doi:10.1073/pnas.0605301104 
27. Crowther G, Napuli A, Thomas A, Chung D, Kovzun K, Leibly D et al (2009) Buffer optimization of thermal melt assays of Plasmodium proteins for detection of small-molecule ligands. J Biomol Screen 14:700-707. doi:10.1177/1087057109335749

28. Ericsson UB, Hallberg BM, DeTitta GT, Dekker N, Nordlund P (2006) Thermofluor-based high-throughput stability optimization of proteins for structural studies. Anal Biochem 357:289-298. doi:10.1016/j. ab.2006.07.027

29. Buscaglia CA, Coppens I, Hol WGJ, Nussenzweig V (2003) Sites of interaction between aldolase and thrombospondin-related anonymous protein in plasmodium. Mol Biol Cell 14:4947-4957. doi:10.1091/mbc. E03-06-0355

30. Kabsch W (1993) Automatic processing of rotation diffraction data from crystals of initially unknown symmetry and cell constants. J Appl Crystallogr 26:795-800. doi: doi:10.1107/S0021889893005588

31. Adams PD, Afonine PV, Bunkóczi G, Chen VB, Echols N, Headd JJ et al (2011) The Phenix software for automated determination of macromolecular structures. Methods 55:94-106. doi:10.1016/j.ymeth.2011.07.005

32. Emsley P, Cowtan K (2004) Coot: model-building tools for molecular graphics. Acta Crystallographica Sect D 60:2126-2132. doi:10.1107/ S0907444904019158

33. Lovell SC, Davis IW, Adrendall WB, de Bakker PIW, Word JM, Prisant MG et al (2003) Structure validation by C alpha geometry: phi, psi and C beta deviation. Proteins 50:437-450

34. Boucher LE, Bosch J (2013) Development of a multifunctional tool for drug screening against Plasmodial protein-protein interactions via surface plasmon resonance. J Mol Recognit 26:496-500. doi:10.1002/jmr.2292

35. Guerreiro-Cacais AO, Levitskaya J, Levitsky V (2010) B cell receptor triggering sensitizes human B cells to TRAIL-induced apoptosis. J Leukoc Biol 88:937-945. doi:10.1189/jlb.0510246

36. Koopman G, Reutelingsperger CP, Kuijten GA, Keehnen RM, Pals ST, van Oers MH (1994) Annexin V for flow cytometric detection of phosphatidylserine expression on B cells undergoing apoptosis. Blood 84:1415-1420

37. Vermes I, Haanen C, Steffens-Nakken H, Reutelingsperger C (1995) A novel assay for apoptosis. Flow cytometric detection of phosphatidylserine expression on early apoptotic cells using fluorescein labelled Annexin V. J Immunol Methods 184:39-51

38. Sattabongkot J, Yimamnuaychoke N, Leelaudomlipi S, Rasameesoraj M, Jenwithisuk R, Coleman RE et al (2006) Establishment of a human hepatocyte line that supports in vitro development of the exo-erythrocytic stages of the malaria parasites Plasmodium falciparum and P. vivax. Am J Trop Med Hyg 74:708-715

39. Coppi A, Cabinian M, Mirelman D, Sinnis P (2006) Antimalarial activity of allicin, a biologically active compound from garlic cloves. Antimicrob Agents Chemother 50:1731-1737

40. Kortagere S, Welsh WJ, Morrisey JM, Daly T, Ejigiri I, Sinnis P et al (2010) Structure-based design of novel small-molecule inhibitors of Plasmodium falciparum. J Chem Inf Model 50:840-849

41. Kumar KA, Oliveira GA, Edelman R, Nardin E, Nussenzweig V (2004) Quantitative Plasmodium sporozoite neutralization assay (TSNA). J Immunol Methods 292:157-164. doi:10.1016/j.jim.2004.06.017

42. Bruña-Romero O, Hafalla JC, González-Aseguinolaza G, Sano G, Tsuji M, Zavala F (2001) Detection of malaria liver-stages in mice infected through the bite of a single Anopheles mosquito using a highly sensitive real-time PCR. Int J Parasitol 31:1499-1502

43. Cardozo T, Abagyan R (2005) Druggability of SCF ubiquitin ligaseprotein interfaces. Methods Enzymol 399:634-653. doi:10.1016/ S0076-6879(05)99042-3

44. Bottegoni G, Kufareva I, Totrov M, Abagyan R (2008) A new method for ligand docking to flexible receptors by dual alanine scanning and refinement (SCARE). J Comput Aided Mol Des 22:311-325. doi:10.1007/ s10822-008-9188-5

45. Pal-Bhowmick I, Andersen J, Srinivasan P, Narum DL, Bosch J, Miller LH (2012) Binding of aldolase and glyceraldehyde-3-phosphate dehydrogenase to the cytoplasmic tails of Plasmodium falciparum merozoite Duffy binding-like and reticulocyte homology ligands. MBio. doi:10.1128/mBio.00292-12

46. Shen B, Sibley LD (2014) Toxoplasma aldolase is required for metabolism but dispensable for host-cell invasion. Proc Natl Acad Sci USA 111:35673572. doi:10.1073/pnas.1315156111

47. Diaz SA, Martin SR, Grainger M, Howell SA, Green JL, Holder AA (2014) Plasmodium falciparum aldolase and the C-terminal cytoplasmic domain of certain apical organellar proteins promote actin polymerization. Mol Biochem Parasitol 197:9-14. doi:10.1016/j.molbiopara.2014.09.006

48. Boucher LE, Bosch J (2015) The apicomplexan glideosome and adhesinsstructures and function. J Struct Biol 190:93-114. doi:10.1016/j.jsb.2015.02.008

49. Gamo F-J, Sanz LM, Vidal J, Cozar Cd, Alvarez E, Lavandera J-L et al (2010) Thousands of chemical starting points for antimalarial lead identification. Nature 465:305-310. doi:10.1038/nature09107

50. Guiguemde WA, Shelat AA, Bouck D, Duffy S, Crowther GJ, Davis PH et al (2010) Chemical genetics of Plasmodium falciparum. Nature 465:311-315

51. Poirot E, Skarbinski J, Sinclair D, Kachur SP, Slutsker L, Hwang J (2013) Mass drug administration for malaria. Cochrane Database Syst Rev 12:CD008846. doi:10.1002/14651858.CD008846.pub2

52. Chardin P, McCormick F (1999) Brefeldin A: the advantage of being uncompetitive. Cell 97:153-155

53. Manfredi JJ, Parness J, Horwitz SB (1982) Taxol binds to cellular microtubules. J Cell Biol 94:688-696

54. Peyroche A, Antonny B, Robineau S, Acker J, Cherfils J, Jackson CL (1999) Brefeldin A acts to stabilize an abortive ARF-GDP-Sec7 domain protein complex: involvement of specific residues of the Sec7 domain. Mol Cell 3:275-285

55. Agrawal RK, Penczek P, Grassucci RA, Frank J (1998) Visualization of elongation factor $\mathrm{G}$ on the Escherichia coli 70 S ribosome: the mechanism of translocation. Proc Natl Acad Sci USA 95:6134-6138

56. Bollag DM, McQueney PA, Zhu J, Hensens O, Koupal L, Liesch J et al (1995) Epothilones, a new class of microtubule-stabilizing agents with a taxol-like mechanism of action. Cancer Res 55:2325-2333

57. Jennewein S, Croteau R (2001) Taxol: biosynthesis, molecular genetics, and biotechnological applications. Appl Microbiol Biotechnol 57:13-19

58. Schiff PB, Fant J, Horwitz SB (1979) Promotion of microtubule assembly in vitro by taxol. Nature 277:665-667

59. Schiff PB, Horwitz SB (1981) Taxol assembles tubulin in the absence of exogenous guanosine 5'-triphosphate or microtubule-associated proteins. Biochemistry 20:3247-3252

60. Ivery MT (2000) Immunophilins: switched on protein binding domains? Med Res Rev 20:452-484

61. Stark H, Rodnina MV, Rinke-Appel J, Brimacombe R, Wintermeyer W, van Heel M (1997) Visualization of elongation factor Tu on the Escherichia coli ribosome. Nature 389:403-406. doi:10.1038/38770

62. Cochran AG (2000) Antagonists of protein-protein interactions. Chem Biol 7:R85-R94

63. Pomel S, Luk FCY, Beckers CJM, Soldati-Favre D (2008) Host cell egress and invasion induce marked relocations of glycolytic enzymes in Toxoplasma gondii tachyzoites. PLoS Pathog 4:e1000188. doi:10.1371/journal.ppat.1000188

64. Starnes GL, Coincon M, Sygusch J, Sibley LD (2009) Aldolase is essential for energy production and bridging adhesin-actin cytoskeletal interactions during parasite invasion of host cells. Cell Host Microbe 5:353-364. doi:10.1016/j.chom.2009.03.005

65. Bosch J, Turley S, Daly TM, Bogh SM, Villasmil ML, Roach C et al (2006) Structure of the MTIP-MyoA complex, a key component of the malaria parasite invasion motor. Proc Natl Acad Sci USA 103:4852-4857. doi:10.1073/pnas.0510907103

66. Bosch J, Turley S, Roach CM, Daly TM, Bergman LW, Hol WG (2007) The closed MTIP-myosin A-tail complex from the malaria parasite invasion machinery. J Mol Biol 372:77-88. doi:10.1016/j.jmb.2007.06.016

67. Turley S, Khamrui S, Bergman LW, Hol WG (2013) The compact conformation of the Plasmodium knowlesi myosin tail interacting protein MTIP in complex with the C-terminal helix of myosin A. Mol Biochem Parasitol 190:56-59. doi:10.1016/j.molbiopara.2013.06.004

68. Douse CH, Maas SJ, Thomas JC, Garnett JA, Sun Y, Cota E et al (2014) Crystal structures of stapled and hydrogen bond surrogate peptides targeting a fully buried protein-helix interaction. ACS Chem Biol 9:2204-2209. doi:10.1021/cb500271c

69. Douse CH, Vrielink N, Wenlin Z, Cota E, Tate EW (2015) Targeting a dynamic protein-protein interaction: fragment screening against the malaria myosin A motor complex. ChemMedChem 10:134-143. doi:10.1002/cmdc.201402357

70. Lipinski CA, Lombardo F, Dominy BW, Feeney PJ (2001) Experimental and computational approaches to estimate solubility and permeability in drug discovery and development settings. Adv Drug Deliv Rev 46:3-26

71. Vida (2015) http://www.eyesopen.com. Accessed 11 June 2015

72. Povray (2015) http://www.povray.com. Accessed 11 June 2015 